\title{
Dominio y rango para funciones elementales de una variable: un problema generado en diversos estudiantes de Ciencias e Ingeniería
}

\author{
Domain and range for elementary functions of a variable: a problem generated in various \\ science and engineering students
}

\section{Domínio e intervalo para funções elementares de uma variável: um problema gerado em vários estudantes de Ciências e Engenharia}

\author{
Andrés Mauricio Nieves Chacón ${ }^{1}$ \\ Universidade Estadual de Santa Cruz (UESC), Ilhéus, BA, Brasil \\ https://orcid.org/0000-0002-8929-5890, $\Theta$ http://lattes.cnpq.br/3832076089281458
}

\begin{abstract}
Resumen: En diversos problemas que surgen al interior de las Ciencias e Ingeniería, los estudiantes deben modelar o reconocer funciones que intervienen en su desarrollo y solución, identificando el papel que cumplen tanto los parámetros, también llamados constantes del problema, como los valores cuyas incógnitas asociadas constituyen una función válida en el conjunto de los números reales. Estos valores son conocidos como dominio y rango, indexados por las incógnitas que reciben el nombre de: variable independiente y variable dependiente, respectivamente. En este artículo se proponen algunos casos que pueden ser abordados por estudiantes universitarios en los temas previos al Cálculo Diferencial e Integral y que servirán para posteriores análisis que respectan a esta misma línea de estudio o áreas afines, acompañados de diferentes técnicas de solución provenientes de la Matemática fundamental.
\end{abstract}

Palabras clave: Funciones; Dominio y Rango; Variable Independiente; Variable Dependiente; Matemática Fundamental.

\begin{abstract}
In the several problems that appear in the scope of Science and Engineering, students must model or recognize functions that intervene in their development and solution, identifying the function fulfilled by both the parameters, also called problem constants, like the values whose associated unknowns constitute the valid function in the set of real numbers. These values are known like domain and range, indexed by the unknowns who are called: independent and dependent variable, respectively. This article proposes some cases that can be addressed by university students in topics prior to Differential and Integral Calculus and which will be used for later analysis on this same line of study or related areas, accompanied by different techniques approached since fundamental Mathematics.
\end{abstract}

Keywords: Functions; Domain and Range; Independent Variable; Dependent Variable; Fundamental Mathematic.

Resumo: Nos vários problemas que surgem nas Ciências e na Engenharia, os alunos devem modelar ou reconhecer funções que intervêm no seu desenvolvimento e solução, identificando o papel desempenhado tanto pelos parâmetros, também chamados constantes do problema, como pelos valores cujas incógnitas associadas constituem um função válida no conjunto de números reais. Esses valores são conhecidos como domínio e intervalo, indexados pelas incógnitas que são chamadas: variável independente e variável dependente, respectivamente. Este artigo propõe alguns casos que podem ser abordados por estudantes universitários nos tópicos anteriores ao Cálculo Diferencial e Integral e que serão utilizados para análises posteriores sobre esta mesma linha de estudo ou áreas afins, acompanhadas por diferentes técnicas de solução da Matemática fundamental.

Palavras-chave: Funções; Domínio e Intervalo; Variável Independente; Variável Dependente; Matemática Fundamental.

Data de submissão: 25 de junho de 2021.

Data de aprovação: 8 de outubro de 2021.

\footnotetext{
1 Currículum sucinto: Ingeniero Industrial pela Universidad del Valle, Colômbia. Mestrando do Programa de Pós Graduação em Modelagem Computacional em Ciência e Tecnologia pela Universidade Estadual de Santa Cruz, ilhéus-Bahia. Contribución del autor: Conceituação, Escrita - Primeira Redação, Escrita - Revisão e Edição, Investigação. Contacto: amnchacon@uesc.br.
} 


\section{Introducción}

El estudio del cálculo diferencial e integral requiere no solo de una correcta capacidad de análisis para enfrentar los problemas propuestos en diversos textos académicos, también demanda las bases que se adquieren desde la matemática fundamental, la cual se compone de la aritmética, la lógica y conjuntos, el álgebra básica, la geometría clásica y analítica, la trigonometría y la introducción al cálculo. Para empezar a entender los primeros temas que el cálculo presenta con sus peculiares características, es necesario comprender de manera acertada el concepto de función y su analogía con el entorno, puesto que:

En diversos campos de la actividad humana, se presentan relaciones que existen entre un conjunto de unos objetos y otro conjunto de otros objetos. Gráficas, cartogramas, curvas, tablas, fórmulas, encuestas en la opinión pública, etc. son familiares a todo aquel que lee los periódicos. En realidad, se trata de puros artificios usados para describir relaciones especiales en forma cuantitativa. Los matemáticos consideran como funciones algunos tipos de estas relaciones (APÓSTOL,1984, p. 61).

De cierta forma, las funciones describen las situaciones reales (PÉREZ GONZÁLES, 2008) como por ejemplo el salario que devenga una persona al depender de la cantidad de horas que trabaje (LEITHOLD, 1998), el área de un circulo que depende de la variabilidad de su radio o la relación que existe entre la temperatura de ebullición del agua y su altitud según el nivel del mar (ADAMS, 2009). Cabe resaltar que al traer a colación el concepto de "relación", este no puede ser confundido con el concepto de "función", puesto que una función $\mathrm{f}(\mathrm{x})$ se puede empezar a definir pensando en el caso de que, al poseer una máquina se produce un valor de salida $\mathrm{f}(\mathrm{x})$, para cada valor de x que sea asignado (ADAMS, 2009), con mayor precisión se puede introducir el concepto de función teniendo en cuenta dos propiedades (CRIBEIRO; MADRID; PRAGA, 2013) que parten del hecho de tener dos conjuntos. Uno de estos recibe el nombre de conjunto de partida, más conocido como dominio, y el segundo recibe el nombre de conjunto de llegada, más conocido como rango, por tanto, la primera propiedad establece que todos los elementos del conjunto de partida, siendo estos aquellos que componen todos los posibles valores que puede tomar la variable independiente, deben estar relacionados con algún elemento del conjunto de llegada. Estos elementos pertenecen a lo que es conocido como variable dependiente, de esta manera, la segunda propiedad dispone que a cada elemento del conjunto de partida le corresponde un único elemento del conjunto de llegada. Por tanto, la "relación" se diferencia de la "función" en el hecho de que en esta se establece una correspondencia de $x_{i}$ elementos del conjunto de partida con $y_{i}$ elementos del conjunto de llegada, sin discutir si cada $\mathrm{x}_{\mathrm{i}}$ posee una única imagen en $\mathrm{y}_{\mathrm{i}}$. Gráficamente, se puede determinar con facilidad las diferencias que posuen ambos conceptos usando el criterio de la recta vertical (GOODMAN; HIRSCH, 1996) y se puede enunciar el clásico ejemplo de una de las tantas curvas hipotrocoides, específicamente la hipocicloide de cuatro 
cúspides, relación que para un caso particular puede ser expresada en su forma cartesiana como $\sqrt[3]{\mathrm{x}^{2}}+\sqrt[3]{\mathrm{y}^{2}}=2$. Sin ahondar en el cálculo de su dominio y rango, su representación gráfica en la Figura 1 muestra que, por medio del criterio de la recta vertical para $\mathrm{x}=1$ existen dos imágenes; por tanto, la curva no es función sino una relación, a diferencia del caso particular de una función logarítmica que se puede expresar como $\mathrm{f}(\mathrm{x})=\log _{5}\left(\mathrm{x}^{2}+1\right)$ cuya representación gráfica se muestra en la Figura 2.

Figura 1 - Representación gráfica del caso particular de una hipocicloide de cuatro cúspides

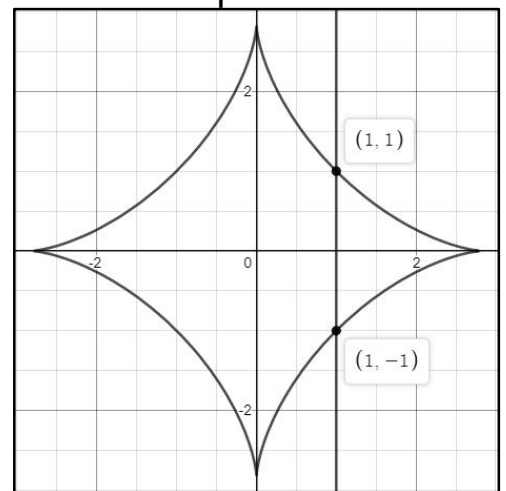

Fuente: Elaborado en la calculadora gráfica Desmos Graphing.

Figura 2 - Representación gráfica del caso particular de una función logarítmica

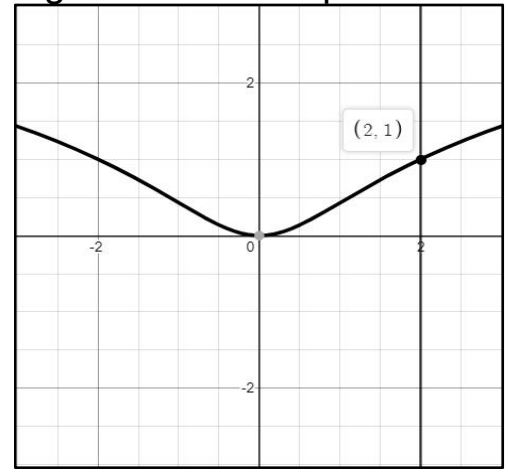

Fuente: Elaborado en la graficadora Desmos Graphing.

La función f de la Figura 2 es decreciente en $(-\infty, 0]$ y creciente en $[0, \infty)$ y al igual que en la curva hipocicloide, por medio del criterio de la recta vertical se evidencia que para $\mathrm{x}=2$ existe una única imagen, como f es decreciente $\forall \mathbb{R}^{-}$y creciente $\forall \mathbb{R}^{+}$, se garantiza que al aplicar dicho criterio por medio de cualquier recta $\mathrm{x}= \pm \mathrm{a}$, siendo \pm a una constante, siempre se cumplirá que esta tenga una única imagen. Este criterio debe aplicarse en toda la extensión del dominio en caso de que se desee verificar gráficamente si es o no una función. De esta manera se puede deducir que las funciones son relaciones debido a que existe una correspondencia de $\mathrm{x} \rightarrow \mathrm{f}(\mathrm{x})$; $\sin$ embargo, las relaciones no necesariamente son funciones. Es importante que el estudiante tenga claro desde un comienzo qué implica el concepto de función con su respectivo alcance, debido a que este trae consigo los dos ejes temáticos que sustentan el presente estudio. El cálculo del dominio y rango de una función se ha convertido en un problema para muchos estudiantes debido 
al análisis particular que se debe aplicar en cada caso propuesto. Por esta razón, en este artículo se plantean ejemplos que desarrollan conceptos y procedimientos completos provenientes de la matemática fundamental, siendo esta la base sólida que traen consigo diferentes axiomas, teoremas o enunciados que ayudan a obtener resultados satisfactorios en la resolución de ejercicios.

\section{Estudios previos frente a la asimilación del concepto de función y sus componentes en los estudiantes}

Diversas investigaciones que se pueden ver en Lupo (2005), Prada Nuñez, Hernández Suárez y Ramírez Leal (2016) y Vanegas Macías y Escalona Fuenmayor (2013), en las que se han evaluado cómo el estudiante aborda el concepto de función y sus implicaciones, denotan que ha existido cierta dificultad para comprender y asimilar la definición con éxito, pues si no se comprende en principio qué es una función y lo que su alcance atañe, el discernimiento de los temas posteriores significará una tarea aún más compleja, tales temas representan el complemento necesario para que el desarrollo del concepto sea cada vez más integral. Muchos estudiantes muestran cierto temor a la hora de enfrentarse a los temas propuestos en los cursos de matemática, puesto que esta ha sido concebida como una ciencia abstracta (VALLE CASTAÑEDA; CASTILLO ESTRELLA; CAMEJO PUENTES, 2018), difícilmente digerible; es necesario que los profesores instauren una metodología que acoja y solucione las dudas por un camino simple, independientemente de que posean estudios pedagógicos o no, cuyo aporte sea que el tema se convierta en un foco en el que la indagación conlleve a escudriñar ejercicios cada vez más exigentes y no que esta sustente el estancamiento del estudiante por no entender el concepto en toda su extensión. Los estudiantes de Ciencias e Ingeniería que se enfrentan a su primer curso de Cálculo, traen consigo la enseñanza impartida en las instituciones educativas o colegios de los cuales provienen (a no ser que su programa académico los nivele con la matemática fundamental) por ende, el docente universitario puede suponer que las bases están claras para empezar los estudios sólidos que su curso a cargo demanda. Desde esta óptica, también han existido estudios (CEBALLOS URREGO; LÓPEZ MONSALVE, 2003) en los que se proponen modelos como los de Van Hiele aplicados en diversos países europeos, que básicamente proporcionan seguir una serie de niveles que van desde una pre-descripción del tema a exponer en las aulas, con el fin de contrastar la idea que previamente tiene sembrada el estudiante, hasta el nivel de deducción formal con base en el reconocimiento, el análisis, la clasificación y el establecimiento de relaciones en los ítems que anteriormente el expositor ha tratado. También se han usado recursos tecnológicos (ROUMIEU, 2014) de manera que si analíticamente no se logra alcanzar el nivel de concepción que el tema requiere, entonces las herramientas gráficas, como facilitadoras visuales de expansión en la compresión, ayudan a que 
las definiciones y procedimientos rigurosos sean más asequibles y digeribles; no siempre es recomendable usar de forma permanente las herramientas gráficas (tecnológicamente hablando) en un curso como el de Cálculo Diferencial, si bien es cierto, estas ayudan a desarrollar procedimientos y a entender lo que en ciertas ocasiones es difícil plasmar en una clase ocasional con papel y lápiz, no porque sea complejo, sino porque puede convertirse en un trabajo muy dispendioso. Este tipo de herramienta puede ser usado y es necesario, sin embargo, el estudiante debe tener claro el procedimiento de cómo lo que la graficadora le muestra se puede esbozar en la hoja de trabajo manual, similar a lo que se hace con los algoritmos que luego deben transcribirse a un software especial por medio de algún lenguaje de programación. De esta manera, el alumno entiende cómo realizar pruebas de escritorio, haciendo de cuenta que él mismo es el compilador y que por tanto cuando se traslade la información al programa computacional, entonces entenderá perfectamente lo que el software ejecuta por causa de la misma lógica que este le indexó. Lo anterior se trae a colación puesto que también se han estudiado casos (SANCHEZ GONZÁLEZ; CANTÚ QUINTANILLA; CANTÚ CUENA, 2011) en los cuales, aun teniendo la gráfica de una función cualquiera, al estudiante le ha resultado difícil calcular el dominio o lo ha confundido con el rango, esto para casos puros, probablemente en una situación que provenga de una aplicación real que posea cierto tipo de restricciones, le sea aún más escabroso el análisis. Aun así, en los casos puros las restricciones no son una excepción, como se expondrá más adelante.

\section{Definición formal de función, dominio y rango desde la teoría de conjuntos y explicaciones preliminares}

\subsection{Definiciones}

Teniendo en cuenta lo que hasta este momento se ha contextualizado, es menester empezar a introducir el concepto de función desde una definición más formal, proveniente de la teoría de conjuntos; inclusive, se introdujo que es necesario que exista una relación de correspondencia entre un conjunto de partida, que en este momento se puede llamar Conjunto $\mathrm{A}$ y un conjunto de llegada, que se puede llamar Conjunto B. Al abordar de manera formal la definición de función, dominio y rango, entre otros conceptos que abarcan el presente artículo, se importarán definiciones del texto de Lógica, conjuntos, relaciones y funciones (PÉREZ RAPOSO, 2010), en el que el Profesor Álvaro P. Raposo acopla diversos teoremas y axiomas que se han estudiado y consolidado a lo largo de la historia.

Definición 3.1.1 Una función $\mathrm{f}$ del conjunto $A$ al conjunto $B$ es un subconjunto del producto cartesiano $A \times B$ en el que no hay dos parejas que tengan el mismo primer elemento. El conjunto $A$ se llama inicial, y el conjunto $B$, final y se denotan con el símbolo $f: A \rightarrow B$. 
Definición 3.1.2 El dominio de una función $\mathrm{f}: \mathrm{A} \rightarrow \mathrm{B}$ es el subconjunto de $\mathrm{A}$ elementos relacionados con algún elemento de $\mathrm{B}$. Se denota como $\mathrm{D}(\mathrm{f})$.

$$
D(f)=\{x \in A \mid \exists y \in B,(x, y) \in f\}
$$

En este punto el estudiante puede preguntarse cuál es el significado de (1). En primer lugar, el hecho de que la Definición 3.1.2 afirme que el dominio es un subconjunto de $A$ elementos, lo que se desea exponer consiste en que tales $A$ elementos representan un sector del conjunto de los números reales $\mathbb{R}$, al igual que $B$, siendo $\mathbb{R}$ el conjunto general. Por tanto el dominio puede ser llamado "subconjunto de $\mathbb{R}$ ", aunque en diversos casos $D(f)$ equivale al conjunto completo de todos los números $\mathbb{R}$, como lo es para funciones lineales constantes de la forma $f(x)=c$ u otras funciones lineales de la forma $f(x)=m x+b$, entre otros casos. Las variables que representan el dominio y rango de $f$ son entonces $x$ e $y$, por ende, entender que $x$ pertenece al conjunto de los números reales, de modo que exista un valor correspondiente en $y$ que pertenezca también a dicho conjunto y que ambos generen un par ordenado que pertenezca a $f$, es una condición razonablemente válida, esto traduce lo que (1) propone. De esta misma forma, se define y se entiende el rango de $f$, también llamado contra dominio.

Definición 3.1.3 El contra dominio de una función $\mathrm{f}: \mathrm{A} \rightarrow \mathrm{B}$ es el subconjunto de $\mathrm{B}$ elementos con los que algún elemento de A está relacionado. Se denota como D'(f).

$$
D^{\prime}(f)=\{y \in B \mid \exists x \in A,(x, y) \in f\}
$$

\subsection{Explicación preliminar}

Es necesario introducir una explicación preliminar desde una función sencilla, con el fin de afianzar las definiciones planteadas, por esta razón, se dispone de la siguiente función lineal para calcular su dominio y rango: $f(x)=\frac{a}{b} x-\frac{c}{d},\{a, b, c, d\} \in \mathbb{R} \wedge\{b, d\} \neq 0$.

\subsubsection{Dominio}

Para calcular el dominio de la función propuesta, el estudiante debe preguntarse: ¿Para qué valores de $\mathbf{x}$ se indetermina $\mathbf{f}$ ? ¿Por qué esta pregunta?, Porque si son conocidos los valores de $\mathrm{x}$ para los cuales $\mathrm{f}$ no es válida, se deduce que para los restantes lo será. Esto es similar a una contradicción, puesto que se encuentra la solución por medio de una pregunta contraria a la definición pura de dominio o rango, que denota los valores consistentes para que esta sea definida en el conjunto de los números reales. Si se introduce la función en una calculadora o en una hoja de cálculo por computadora, la pregunta coloquial sería: ¿Para qué valores de $x$ se mostrará el letrero “MATH ERROR” o una de las distintivas notas “\# ¡N/D!?”. 
Para el caso propuesto, ningún valor en el conjunto de los números reales hará que la función se indetermine. Por lo tanto, su dominio será válido $\forall \mathbb{R}$ y puede ser expresado de dos formas igualmente válidas: $\mathrm{D}(\mathrm{f})=\mathbb{R} \circ \mathrm{D}(\mathrm{f})=(-\infty, \infty)$.

\subsubsection{Rango}

La pregunta esencial para calcular el rango de $\mathrm{f}$ es exactamente igual que lo planteado en el título 3.2.1, sólo que se debe expresar la variable independiente $\mathrm{x}$ en términos de la variable dependiente $\mathrm{f}(\mathrm{x})$, es decir, formular la situación de manera que $\mathrm{f}(\mathrm{x}) \rightarrow \mathrm{x}$, con el fin de facilitar el análisis y equiparar la metodología de cálculo. Se procede entonces a expresar x en términos de $f(x)$. Para efectos procedimentales se definirá que $f(x)=y$, de esta manera se tiene que: $\frac{a}{b} x-\frac{c}{d}=$ $\mathrm{y} \therefore \mathrm{x}=\frac{(\mathrm{bd}) \mathrm{y}+\mathrm{bc}}{\mathrm{ad}}$. En este orden de ideas, se puede notar que no hay restricción para algún valor de y, en consecuencia, el rango de la función se puede escribir al igual que el dominio como: $D^{\prime}(f)=\mathbb{R} \circ D^{\prime}(f)=(-\infty, \infty)$. Para $x=\frac{(b d) y+b c}{a d}$ es válido aclarar que $a \neq 0$, sin embargo, si en un comienzo a hubiese sido igual a cero siendo $\{b, d\} \neq 0$, entonces $f(x)=-\frac{c}{d}$, la cual se convierte en un caso particular de una función lineal constante, que implica de igual forma que su dominio sea todo el conjunto de los números reales. No obstante, el rango es distinto, debido a que para cualquier valor de $\mathrm{x}, \mathrm{f}(\mathrm{x})$ tomará siempre el mismo valor que es $-\frac{\mathrm{c}}{\mathrm{d}}$, por ende su rango no varía y será la constante misma $D^{\prime} f(x)=-\frac{c}{d}$. Esta explicación preliminar, aunque data de ejemplos relativamente simples, expone la pregunta fundamental para dar solución a cualquier ejercicio de dominio y rango, que en la medida de lo posible pueda ser solucionado por medio de métodos analíticos y/o procedimientos algebraicos ya constituidos; de cierta manera se evidencia que el cálculo del rango posee un plus o característica adicional, y es que aparte de analizarlo con la misma metodología implementada para calcular el dominio, se debe previamente realizar un despeje, en el que si inicialmente se tiene $\mathrm{x} \rightarrow \mathrm{f}(\mathrm{x})$ luego se tenga $\mathrm{f}(\mathrm{x}) \rightarrow \mathrm{x}$, esto implica que, calcularlo posee un grado mayor de dificultad. En muchos casos, dependiendo de qué tan compleja sea la función, es recomendable dibujarla y determinar de manera gráfica su dominio y rango. Para esto es válido usar métodos de análisis gráfico como el cálculo de límites unilaterales, límites infinitos y al infinito que conllevan al cálculo de asíntotas verticales y horizontales, cortes con los ejes coordenados, transformación de funciones, optimización gráfica o análisis de simetría, todo esto según corresponda el caso. Sin embargo, uno de los ejes que respecta a este artículo, trata de no dar mucho uso a los temas en los cuales se ahonde en los procedimientos que el cálculo diferencial provee, con el fin de mostrar la riqueza que poseen las técnicas clásicas previas al cálculo. 


\section{Casos propuestos}

\subsection{Función elemental con factores radicales y exponenciales}

Se propone el siguiente caso para calcular el dominio de la función $\mathrm{g}(\mathrm{x})$, por medio del cual el estudiante debe recurrir a las técnicas algebraicas fundamentales y hacer uso de métodos analíticos para dar solución a las inecuaciones (desigualdades) que puedan generarse.

$$
g(x)=\frac{\sqrt[5]{x} \cdot \sqrt{x^{4}-x^{5}-x^{3}+x^{6}-x^{2}+x}}{\left(12 x^{3}-16 x^{2}-41 x+15\right)\left(2^{\sqrt[3]{x^{2}}}-e^{2}\right)}
$$

Cuando se proponen funciones que contienen diversas características como $\mathrm{g}(\mathrm{x})$, es recomendable analizarlas por partes, es decir: $g(x)=\frac{\sqrt[n]{u_{1}(x)} \cdot \sqrt[m]{u_{2}(x)}}{u_{3}(x) \cdot u_{4}(x)}$. Para cual se establece que: $\mathrm{u}_{1}(\mathrm{x})=\mathrm{x}, \mathrm{u}_{2}(\mathrm{x})=\mathrm{x}^{4}-\mathrm{x}^{5}-\mathrm{x}^{3}+\mathrm{x}^{6}-\mathrm{x}^{2}+\mathrm{x}, \mathrm{u}_{3}(\mathrm{x})=12 \mathrm{x}^{3}-16 \mathrm{x}^{2}-41 \mathrm{x}+15, \mathrm{u}_{4}(\mathrm{x})=2^{\sqrt[3]{\mathrm{x}^{2}}}-\mathrm{e}^{2} \therefore$ e es el número irracional Euler, $\mathrm{n}=5 ; \mathrm{m}=2$. La pregunta usual para iniciar el análisis es: ¿Para qué valores de $\mathrm{x}$ se indetermina $\mathrm{g}$ ? $\mathrm{Si} \mathrm{g}(\mathrm{x})=\frac{\mathrm{p}(\mathrm{x})}{\mathrm{q}(\mathrm{x})}$, entonces $\mathrm{q}(\mathrm{x})$ debe ser distinto de cero, por lo tanto, si $\mathrm{u}_{3}(\mathrm{x}) \cdot \mathrm{u}_{4}(\mathrm{x})=0, \mathrm{~g}(\mathrm{x})$ no será válida, de esta manera, $\mathrm{u}_{3}(\mathrm{x}) \neq 0 \wedge \mathrm{u}_{4}(\mathrm{x}) \neq 0$. Adicional a esto, las expresiones que componen el numerador de la función son de tipo radical, donde $\mathrm{n}$ y m representan los índices de las raíces, impar y par, respectivamente. Como n representa a una raíz impar, $\mathrm{u}_{1}(\mathrm{x})$ no tendrá restricciones, puesto que si $\mathrm{u}_{1}(\mathrm{x}) \leq 0$, este hecho no hará que g sea en inválida $\forall \mathbb{R}$, debido a que las raíces cuyo índice sea impar, serán admitidas $\forall \mathbb{R}^{-}$como lo son $\forall \mathbb{R}^{+}$, por tanto, $\mathrm{u}_{1}(\mathrm{x})$ no será objeto de preocupación para el análisis del cálculo del dominio en $\mathrm{g}(\mathrm{x})$. Lo contrario sucede para las raíces cuyo índice es par, para este caso en concreto, $\mathrm{u}_{2}(\mathrm{x})$ debe ser no negativa, por lo que si $\mathrm{u}_{2}(\mathrm{x})$ es menor que cero, $\mathrm{g}(\mathrm{x})$ no será válida en $\mathbb{R}$, por ende $\mathrm{u}_{2}(\mathrm{x}) \geq 0$. Note que si $\sqrt[m]{u_{2}(x)}$ hubiese sido un factor de $q(x)$, entonces $u_{2}(x) \geq 0$ no sería una expresión correcta para este contexto, puesto que daría permiso a que $\mathrm{q}(\mathrm{x})$ pueda tomar el valor de cero, lo cual hace que $\mathrm{g}(\mathrm{x})$ se indetermine, para tal caso entonces es válido afirmar que $\mathrm{u}_{2}(\mathrm{x})>0$. De esta manera se listan las condiciones generales para las cuales $g(x)$ es consistente: $i . u_{2}(x) \geq 0$ y ii. $\mathrm{u}_{3}(\mathrm{x}) \neq 0 \wedge \mathrm{u}_{4}(\mathrm{x}) \neq 0$.

\subsubsection{Análisis de la condición $i$}

Esta condición consiste en el clásico ejercicio de desigualdades, $\mathrm{u}_{2}(\mathrm{x}) \geq 0$ se traduce como: $x^{4}-x^{5}-x^{3}+x^{6}-x^{2}+x \geq 0$. En este punto, el estudiante se debe preguntar ¿Para qué valores de $\mathrm{x}$ se cumple la desigualdad? Para ello es necesario que $\mathrm{u}_{2}(\mathrm{x})$ sea visualmente más sencilla de analizar. Esto se logra si es posible factorizar el polinomio, para lo cual se recomienda organizar la expresión con el fin de verificar el método que puede ser usado: $x^{6}-x^{5}+x^{4}-x^{3}-$ 
$x^{2}+x \geq 0$. Es aquí donde muchos estudiantes presentan fallas, ya que el error de factorizar de forma inadecuada suele ser común (GAMBOA; CASTILLO; HIDALGO MORA, 2019), puesto que no recuerdan los casos de factorización enseñados en la secundaria o se realizan asociaciones erradas según los factores de descomposición (OLIVAR MOLINA; FLORES LÓPEZ; ALVARADO GONZÁLEZ, 2018). Una investigación como la de (MEJÍA PALOMINO, 2011) expone los errores comunes que presentan estudiantes de un curso específico de secundaria, además de su aporte en la enseñanza del álgebra fundamental. Analizando $\mathrm{u}_{2}(\mathrm{x})$, inicialmente se aplica el factor común monomio de $x: x\left(x^{5}-x^{4}+x^{3}-x^{2}-x+1\right) \geq 0$. Al visualizar detalladamente la expresión, se pueden acoplar tres binomios con el fin de aplicar el factor común por agrupación de términos (también llamado factor común polinomio) y posteriormente de nuevo el factor común monomio: $\mathrm{x}\left[\left(\mathrm{x}^{5}-\mathrm{x}^{4}\right)+\left(\mathrm{x}^{3}-\mathrm{x}^{2}\right)-(\mathrm{x}-1)\right] \geq 0 \quad \therefore \mathrm{x}\left[\mathrm{x}^{4}(\mathrm{x}-1)+\mathrm{x}^{2}(\mathrm{x}-1)-(\mathrm{x}-1)\right] \geq 0$. Como $\mathrm{x}-1$ representa un factor común entre las expresiones que se encuentran dentro de los corchetes, entonces: $x(x-1)\left(x^{4}+x^{2}-1\right) \geq 0$. La nueva traducción de la desigualdad equivale $a: x(x-$ 1) $\left(x^{4}+x^{2}-1\right) \geq 0$, que para efectos prácticos se puede notar como: $\mathrm{v}_{1}(\mathrm{x}) \cdot \mathrm{v}_{2}(\mathrm{x}) \cdot \mathrm{v}_{3}(\mathrm{x}) \geq 0$,respectivamente. ¿En qué casos es posible que este triple producto sea no negativo? a continuación se listan los casos posibles para los cuales cada uno representa un intervalo de solución $\mathrm{I}_{\mathrm{i}}$ :

$$
\begin{array}{ll}
\mathrm{I}_{1}=\mathrm{u}_{2}(\mathrm{x}) \geq 0 \leftrightarrow \mathrm{v}_{1}(\mathrm{x}) \geq 0 \wedge \mathrm{v}_{2}(\mathrm{x}) \geq 0 & \wedge \mathrm{v}_{3}(\mathrm{x}) \geq 0 \\
\mathrm{I}_{2}=\mathrm{u}_{2}(\mathrm{x}) \geq 0 \leftrightarrow \mathrm{v}_{1}(\mathrm{x}) \leq 0 \wedge \mathrm{v}_{2}(\mathrm{x}) \leq 0 & \wedge \mathrm{v}_{3}(\mathrm{x}) \geq 0 \\
\mathrm{I}_{3}=\mathrm{u}_{2}(\mathrm{x}) \geq 0 \leftrightarrow \mathrm{v}_{1}(\mathrm{x}) \leq 0 \wedge \mathrm{v}_{2}(\mathrm{x}) \geq 0 & \wedge \mathrm{v}_{3}(\mathrm{x}) \leq 0 \\
\mathrm{I}_{4}=\mathrm{u}_{2}(\mathrm{x}) \geq 0 \leftrightarrow \mathrm{v}_{1}(\mathrm{x}) \geq 0 \wedge \mathrm{v}_{2}(\mathrm{x}) \leq 0 & \wedge \mathrm{v}_{3}(\mathrm{x}) \leq 0
\end{array}
$$

De estos cuatro intervalos, la solución S estará dada por la diferencia simétrica entre cada uno expresada como $\mathrm{S}=\mathrm{I}_{1} \Delta \mathrm{I}_{2} \Delta \mathrm{I}_{3} \Delta \mathrm{I}_{4}$. Se introduce este concepto debido a que cada intervalo puede contener al menos un valor que satisfaga que $u_{2}(x) \geq 0$ y la idea consiste en incluir todas estas soluciones, esto se obtiene mediante la diferencia simétrica y no la intersección, debido a que en la intersección se pueden excluir muchos valores que no hacen parte de la restricción del dominio de $\mathrm{g}(\mathrm{x})$.

Definición 4.1.1.1 La diferencia simétrica de dos conjuntos $A$ y $B$ es el conjunto formado por los elementos que están en $A$ o están en $B$ excepto los comunes a ambos. Se denota $A \Delta B$ y se puede escribir como

$$
\mathrm{A} \Delta \mathrm{B}=(\mathrm{A} \backslash \mathrm{B}) \cup(\mathrm{B} \backslash \mathrm{A})
$$

$\mathrm{A} \backslash \mathrm{B}$ se traduce como todos los elementos que pertenecen única y exclusivamente al conjunto A. De la misma forma se traduce el conjunto $B \backslash A$. Los valores de $x$ que satisfacen la consistencia en las desigualdades asociadas $a v_{1}(x)$ y $v_{2}(x)$, representan expresiones lineales cuya solución es directa. Como $\mathrm{v}_{1}(\mathrm{x}) \geq 0$ entonces $\mathrm{x} \geq 0$, por tanto, la solución se representa por 
medio del intervalo semiabierto: $[0, \infty)$ y en su forma contraria $v_{1}(x) \leq 0$ se tiene que $x \leq 0$ que significa $(-\infty, 0]$. De la misma forma se resuelve con $v_{2}(x)$, así que para $v_{2}(x) \geq 0 \wedge v_{2}(x) \leq 0$, se tiene que $\mathrm{x}-1 \geq 0$ y $\mathrm{x}-1 \leq 0$, cuyas soluciones son $\mathrm{x} \geq 1$ y $\mathrm{x} \leq 1$, representadas por $[1, \infty)$ y $(-\infty, 1]$, respectivamente. Hasta esta instancia, el estudiante debe entender el uso que tienen los signos de agrupación en la aplicación de intervalos, por ejemplo, si $x \geq 1$ su notación es $[1, \infty)$ y no $(1, \infty)$ puesto que $x=1$ hace parte del intervalo al ser precisamente $x \geq 1$, la opción de $(1, \infty)$ hubiese sido valida si $x>1$. Para $v_{3}(x)$ se requiere un procedimiento especial puesto que se trata de un polinomio de grado $\mathrm{n}>1$ y por tanto no lineal. Con el fin de que el estudiante comprenda la forma en como $v_{3}(x) \geq 0$ y $v_{3}(x) \leq 0$ se solucionan, en primer lugar, se puede pensar en las soluciones anteriores de $\mathrm{v}_{1}(\mathrm{x})$ y $\mathrm{v}_{2}(\mathrm{x})$, considerando que el número que se encuentra al lado derecho de las desigualdades asociadas a ellas representan sus raíces, es decir, aquellos valores para los cuales $v_{1}(x)=0$ y $v_{2}(x)=0$, en otras palabras, si $v_{1}(x)$ y $v_{2}(x)$ se graficasen, tales valores representan los puntos de corte con el eje de las abscisas. El teorema fundamental del álgebra (PÉREZ LLUBERES, 1984) propuesto por Gauss, uno de sus más importantes aportes a la matemática, establece que una función polinómica $\mathrm{f}$ de grado $\mathrm{n}$, donde $\mathrm{n} \neq 0$ posee al menos una raíz real o compleja y si f es expresada en factores lineales de la forma $(x-k)$, entonces $k$ es una raíz de $\mathrm{f}$. Este concepto se introduce con el fin de calcular las raíces de $\mathrm{v}_{3}(\mathrm{x})$. Al hallarlas será posible determinar los intervalos para los cuales $v_{3}(x) \geq 0$ y $v_{3}(x) \leq 0$ evaluando un número $q$ cualquiera que se encuentre dentro del intervalo formado, tal evaluación se denota como v(q). Esto último consiste en otra forma de solucionar una desigualdad de grado $n>1$. Para $\mathrm{v}_{3}(\mathrm{x})=$ $x^{4}+x^{2}-1$, note que es posible realizar un cambio de variable en términos de que $p=x^{2}$, de esta manera $v_{3}(x)$ se transforma $a v_{3}(p)=p^{2}+p-1$, siendo $v_{3}(p)$ un polinomio cuadrático. Para determinar si $\mathrm{v}_{3}(\mathrm{p})$ posee raíces, basta con calcular su discriminante $\mathrm{D}_{\mathrm{v}_{3}(\mathrm{p})}$, para este caso, el discriminante de un polinomio cuadrático equivale al radicando proveniente de la solución de la ecuación cuadrática $\mathrm{ax}^{2}+\mathrm{bx}+\mathrm{c}=0$, para este caso aplicado en $\mathrm{v}_{3}(\mathrm{p})$ se tiene de forma genérica que $a p^{2}+b p+c=0$, para la cual:

$$
\mathrm{p}=\frac{-\mathrm{b} \pm \sqrt{\mathrm{b}^{2}-4 \mathrm{ac}}}{2 \mathrm{a}}=\frac{-\mathrm{b} \pm \sqrt{\mathrm{D}}}{2 \mathrm{a}}
$$

Si $\mathrm{D}>0$ existen dos soluciones no necesariamente distintas. $\mathrm{D}_{\mathrm{v}_{3}(\mathrm{p})}=(1)^{2}-4(1)(-1)=5$. Como $\mathrm{D}_{\mathrm{v}_{3}(\mathrm{p})}>0$, entonces existen dos soluciones para $\mathrm{v}_{3}(\mathrm{p})$, por tanto se tienen dos raíces: $\mathrm{p}=$ $\frac{-(1) \pm \sqrt{5}}{2(1)}=\frac{-1 \pm \sqrt{5}}{2}$. Al ser $p_{1}=\frac{-1+\sqrt{5}}{2}$ y $p_{2}=\frac{-1-\sqrt{5}}{2}, v_{3}(p)$ en factores lineales es $\left(p-p_{1}\right)\left(p-p_{2}\right), \sin$ embargo, como $\mathrm{p}=\mathrm{x}^{2}$ entonces $\mathrm{v}_{3}(\mathrm{x})=\left(\mathrm{x}^{2}+\frac{1-\sqrt{5}}{2}\right)\left(\mathrm{x}^{2}+\frac{1+\sqrt{5}}{2}\right)$. El estudiante debe tener cuidado con el tratamiento de los signos en $\mathrm{v}_{3}(\mathrm{x})$. Para solucionar $\mathrm{v}_{3}(\mathrm{x}) \geq 0$, se calculan las raíces de $\mathrm{v}_{3}(\mathrm{x})$ y se introduce una nueva forma de darle solución a la desigualdad, para esto, primero se 
calculan los valores de $\mathrm{x}$ para los cuales $\mathrm{v}_{3}(\mathrm{x})=0 ; \mathrm{x}^{2}=-\frac{1 \pm \sqrt{5}}{2} \therefore \mathrm{x}= \pm \sqrt{-\frac{1 \pm \sqrt{5}}{2}}$. Se tienen cuatro raíces para $\mathrm{v}_{3}(\mathrm{x})$ :

$$
\begin{aligned}
& x_{1}=\sqrt{\frac{-1+\sqrt{5}}{2}} ; x_{2}=-\sqrt{\frac{-1+\sqrt{5}}{2}} ; \\
& x_{3}=\sqrt{\frac{-1-\sqrt{5}}{2}} ; x_{4}=-\sqrt{\frac{-1-\sqrt{5}}{2}}
\end{aligned}
$$

Sin embargo, $x_{3} y_{x_{4}}$ son imaginarias, por lo cual no se tendrán en cuenta en este análisis, ya que $\quad \mathrm{x}_{3^{\wedge} 4} \notin \mathbb{R} . \quad \mathrm{x}_{3^{\wedge} 4}= \pm \sqrt{\frac{-1-\sqrt{5}}{2}}= \pm \sqrt{\frac{1+\sqrt{5}}{2}} \cdot \sqrt{-1}= \pm \sqrt{\frac{1+\sqrt{5}}{2}} \mathrm{i}$. Teniendo en cuenta las raíces reales halladas para $\mathrm{v}_{3}(\mathrm{x})$, al evaluar un valor $\mathrm{q}$, que para este caso se toman $\mathrm{q}_{1}=-$ $2, \mathrm{q}_{2}=0, \mathrm{q}_{3}=2$, que deben pertenecer estrictamente a cada uno de los tres intervalos en cuestión y distinto a las raíces, se tiene que:

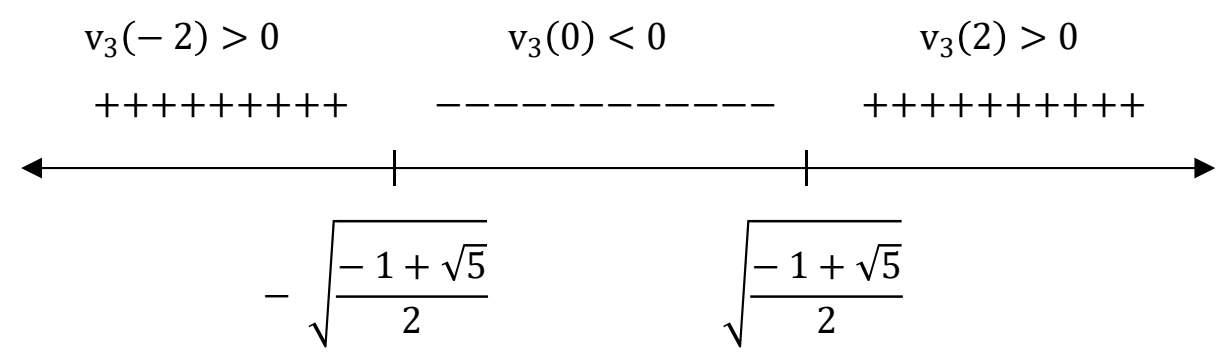

Note que $\pm \sqrt{\frac{-1+\sqrt{5}}{2}} \approx \pm 0.786$, esto se apunta como guía para saber qué valor de q escoger en cada intervalo. De esta manera: $v_{3}(x) \geq 0 \forall x \leq-\sqrt{\frac{-1+\sqrt{5}}{2}} \wedge \forall x \geq \sqrt{\frac{-1+\sqrt{5}}{2}}$; es decir: $\mathrm{v}_{3}(\mathrm{x}) \geq 0 \leftrightarrow \mathrm{x} \in\left\{\left(-\infty,-\sqrt{\frac{-1+\sqrt{5}}{2}}\right] \cup\left[\sqrt{\frac{-1+\sqrt{5}}{2}}, \infty\right)\right\} \mathrm{y} \mathrm{v}_{3}(\mathrm{x}) \leq 0 \quad \forall \mathrm{x} \in\left[-\sqrt{\frac{-1+\sqrt{5}}{2}}, \sqrt{\frac{-1+\sqrt{5}}{2}}\right]$.

El tratamiento realizado para dar solución $\mathrm{a}_{3}(\mathrm{x}) \geq 0$ y $\mathrm{v}_{3}(\mathrm{x}) \leq 0$ consistió en aplicar el clásico método tabular en el cual participan los conocidos valores de prueba, inclusive, $\mathrm{u}_{2}(\mathrm{x}) \geq 0$ pudo ser resuelta utilizando este método para todas las raíces de $u_{2}(x)$, lo cual reduce el procedimiento y hace que se convierta en una desigualdad más simple. Sin embargo, se introduce solo hasta este punto con el fin de concientizar al estudiante acerca de qué métodos analíticos implican, lo cual al conocerlos hacen de la habilidad matemática una herramienta más integral, y no solo de conocer el camino más corto sin interiorizar el razonamiento del mismo. Al retomar los intervalos $\mathrm{I}_{\mathrm{i}}$ que darán la solución $\mathrm{S}$ de $\mathrm{u}_{2}(\mathrm{x}) \geq 0$, se tiene que:

$$
I_{1}=[0, \infty) \cap[1, \infty) \cap\left\{\left(-\infty,-\sqrt{\frac{-1+\sqrt{5}}{2}}\right] \cup\left[\sqrt{\frac{-1+\sqrt{5}}{2}}, \infty\right)\right\}=[1, \infty)
$$




$$
\begin{gathered}
I_{2}=(-\infty, 0] \cap(-\infty, 1] \cap\left\{\left(-\infty,-\sqrt{\frac{-1+\sqrt{5}}{2}}\right] \cup\left[\sqrt{\frac{-1+\sqrt{5}}{2}}, \infty\right)\right\}=\left(-\infty,-\sqrt{\frac{-1+\sqrt{5}}{2}}\right] \\
I_{3}=(-\infty, 0] \cap[1, \infty) \cap\left[-\sqrt{\frac{-1+\sqrt{5}}{2}}, \sqrt{\frac{-1+\sqrt{5}}{2}}\right]=\varnothing \text { (Vacio) } \\
I_{4}=[0, \infty) \cap(-\infty, 1] \cap\left[-\sqrt{\frac{-1+\sqrt{5}}{2}}, \sqrt{\frac{-1+\sqrt{5}}{2}}\right]=\left[0, \sqrt{\frac{-1+\sqrt{5}}{2}}\right]
\end{gathered}
$$

Note que los intervalos $I_{i}$ fueron expresados a modo de conjunción, para lo cual es necesario recordar que la intersección de dos conjuntos representa el conjunto formado por los elementos que se encuentran en ambos, es decir, aquellos elementos comunes en cada conjunto denotados por $\mathrm{A} \cap \mathrm{B}$, para el caso en cuestión la intersección debe ser simultánea en cada tripleta de conjuntos. De esta manera, la diferencia simétrica $\mathrm{S}$ por la Definición 4.1.1.1 es: $\mathrm{S}=$ $\mathrm{I}_{1} \Delta \mathrm{I}_{2} \Delta \mathrm{I}_{3} \Delta \mathrm{I}_{4}$

$$
\begin{aligned}
S= & {[1, \infty) \Delta\left(-\infty,-\sqrt{\frac{-1+\sqrt{5}}{2}}\right] \Delta\left[0, \sqrt{\frac{-1+\sqrt{5}}{2}}\right]=} \\
& \left(-\infty,-\sqrt{\frac{-1+\sqrt{5}}{2}}\right] \cup\left[0, \sqrt{\frac{-1+\sqrt{5}}{2}}\right] \cup[1, \infty)
\end{aligned}
$$

Racionalizando denominadores y organizando la expresión al interior de las raíces:

$$
S=\left(-\infty,-\frac{\sqrt{2}}{2} \cdot \sqrt{\sqrt{5}-1}\right] \cup\left[0, \frac{\sqrt{2}}{2} \cdot \sqrt{\sqrt{5}-1}\right] \cup[1, \infty)
$$

\subsubsection{Análisis de la condición ii}

Esta condición parte del hecho de que $\mathrm{u}_{3}(\mathrm{x}) \neq 0 \wedge \mathrm{u}_{4}(\mathrm{x}) \neq 0$, por tanto, se deben calcular todos los valores de $x$ para los cuales $u_{3}(x)=0 \wedge u_{4}(x)=0$ y excluirlos del intervalo final de solución. Para $u_{3}(x)$ se sabe que $u_{3}(x)=12 x^{3}-16 x^{2}-41 x+15$. Gauss también extiende su teorema fundamental y lo complementa con el teorema de las raíces racionales, que establece que una ecuación polinómica de la forma:

$$
c_{n} x^{n}+c_{n-1} x^{n-1}+c_{n-2} x^{n-2}+\cdots+c_{0}=0
$$

Donde $c_{n} \wedge c_{0} \in \mathbb{Z}$, ambos distintos de cero y se sustenta que $x$ posee soluciones de la forma: $x=\frac{a}{b}, b \neq 0$. Siendo $a \wedge b$ divisores de $c_{0} \wedge c_{n}$, respectivamente. $u_{3}(x)$ puede ser expresado en factores lineales $\left(x-\frac{a}{b}\right)$, para ello primero se deben calcular todos los posibles $\frac{a}{b}$ : $\mathrm{D}_{\mathrm{c}_{0}}=\mathrm{D}_{15}=\{ \pm 1, \pm 3, \pm 5, \pm 15\} \quad$ y $\mathrm{D}_{\mathrm{c}_{\mathrm{n}}}=\mathrm{D}_{12}=\{ \pm 1, \pm 2, \pm 3, \pm 4, \pm 6, \pm 12\}$. De esta 
manera: $\frac{\mathrm{a}}{\mathrm{b}}=\left\{ \pm 1, \pm \frac{1}{2}, \pm \frac{1}{3}, \pm \frac{1}{4}, \pm \frac{1}{6}, \pm \frac{1}{12}, \pm 3, \pm \frac{3}{2}, \cdots, \pm \frac{15}{12}\right\}$. Luego se debe realizar una división polinómica, cuyo divisor será $\left(\mathrm{x}-\frac{\mathrm{a}}{\mathrm{b}}\right)$, para esto se debe probar cada $\frac{\mathrm{a}}{\mathrm{b}}$ y con el fin de simplificar el proceso se hará uso de la regla de división sintética propuesta por Paolo Ruffini, que representa una alternativa a la división polinómica usando como divisor un factor lineal; Para esta regla, el éxito se encuentra cuando el residuo de la división es cero, para lo cual se tiene que:

$$
\operatorname{Ddo}(\mathrm{x})=\operatorname{Div}(\mathrm{x}) \cdot \operatorname{Cte}(\mathrm{x})+\mathrm{r}
$$

Donde $\operatorname{Ddo}(\mathrm{x})$ es el polinomio que representa el dividendo, $\operatorname{Div}(\mathrm{x})$ es el polinomio que representa el divisor y $\mathrm{Cte}(\mathrm{x})$ es el cociente, $\mathrm{r}$ es un factor numérico constante que viene a ser en realidad el residuo, por tanto, este debe ser cero para que Ddo(x) pueda ser expresado como un producto de polinomios sin factores constantes externos a dicho producto. Note que Ddo(x) presenta la forma clásica utilizada para probar la división, inclusive, enseñada por los profesores de la básica primaria, no a nivel de polinomios respecto a una variable, sino con los números naturales que a esa etapa se estudian, es la llamada prueba de la división. El algoritmo de Ruffini aplicado en $\mathrm{u}_{3}(\mathrm{x})$ para $\mathrm{x}=\frac{1}{3}$ resuelve:

Cuadro 1 - Algoritmo de Ruffini
\begin{tabular}{|rrrc|c|}
\hline 12 & -16 & -41 & 15 & $\frac{1}{3}$ \\
4 & -4 & -15 & \\
\hline 12 & -12 & -45 & $\begin{array}{c}0 \\
\text { Residuo }\end{array}$ & \\
\hline
\end{tabular}

Fuente: Elaboración propia.

De esta manera, $\mathrm{u}_{3}(\mathrm{x})=\left(\mathrm{x}-\frac{1}{3}\right)\left(12 \mathrm{x}^{2}-12 \mathrm{x}-45\right)$ y para $12 \mathrm{x}^{2}-12 \mathrm{x}-45$ se puede aplicar de nuevo el anterior algoritmo o calcular las raíces usando la solución de la ecuación cuadrática, sin embargo, con el fin de recordar que existe el método de factorización cuya composición consiste en el trinomio de la forma $\mathrm{k}^{2 \mathrm{n}} \pm \mathrm{bk}^{\mathrm{n}} \pm \mathrm{c}$, note que $\mathrm{k}=2 \mathrm{x}: 12 \mathrm{x}^{2}-12 \mathrm{x}-45=3 \cdot\left((\mathrm{k})^{2}-\right.$ $2(\mathrm{k})-15)=3 \cdot(\mathrm{k}-5)(\mathrm{k}+3)=3 \cdot(2 \mathrm{x}-5)(2 \mathrm{x}+3)$. De esta manera $\mathrm{u}_{3}(\mathrm{x})=\left(\mathrm{x}-\frac{1}{3}\right) \cdot 3 \cdot(2 \mathrm{x}-$ 5) $(2 x+3)$, simplificando: $u_{3}(x)=(3 x-1)(2 x-5)(2 x+3)$. Para $u_{3}(x)=0$ se obtienen las raíces: $\mathrm{x}_{1}=\frac{1}{3}, \mathrm{x}_{2}=\frac{5}{2}, \mathrm{x}_{3}=-\frac{3}{2}$. De la misma forma se analiza para $\mathrm{u}_{4}(\mathrm{x})=0$, que contiene un término exponencial. El estudiante debe recordar que para dar solución a $u_{4}(x)=0$, es fundamental conocer la propiedad logarítmica:

$$
\log _{a} b^{n}=n \log _{a} b
$$


Por tanto: $2^{\sqrt[3]{\mathrm{x}^{2}}}-\mathrm{e}^{2}=0 \quad \therefore \quad \mathrm{x}= \pm \sqrt{\left(\frac{2}{\ln 2}\right)^{3}}= \pm \sqrt{\frac{8}{\ln ^{3} 2}}$, simplificando $\mathrm{y}$ racionalizando: $\mathrm{x}= \pm$ $\frac{2 \sqrt{2 \ln 2}}{\ln ^{2} 2}$; Note que $\ln \mathrm{e}=1$, puesto que el logaritmo natural se encuentra en base e, es decir $\ln \mathrm{e}=$ $\log _{\mathrm{e}}$ e y por la propiedad $\log _{\mathrm{a}} \mathrm{a}=1$, entonces $\ln \mathrm{e}=1$. Cabe resaltar que no es una camisa de fuerza aplicar el método de Ruffini, pues otra alternativa usada es el método de Cardano (OOSTRA, 2008), en donde de manera analítica, usando procedimientos algebraicos más rigurosos, se desenvuelven una serie de ecuaciones para las cuales, haciendo las sustituciones correctas se obtiene el resultado.

\subsubsection{Dominio de la función $\mathrm{g}(\mathrm{x})$}

El dominio de la función $\mathrm{g}(\mathrm{x})$ serán todos los valores reales de x para los cuales se cumpla que $x \in S \quad y$ a su vez $x \neq\left\{-\frac{2 \sqrt{2 \ln 2}}{\ln ^{2} 2},-\frac{3}{2}, \frac{1}{3}, \frac{5}{2}, \frac{2 \sqrt{2 \ln 2}}{\ln ^{2} 2}\right\}$. Como $S=\left(-\infty,-\frac{\sqrt{2}}{2} \cdot \sqrt{\sqrt{5}-1}\right] \cup$ $\left[0, \frac{\sqrt{2}}{2}(\sqrt{\sqrt{5}-1})\right] \cup[1, \infty)$, entonces: $\mathrm{D}(\mathrm{g}(\mathrm{x}))=\left\{\mathrm{x} \in \mathbb{R} \mid \mathrm{x} \in \mathrm{S} \wedge \mathrm{x} \neq\left\{-\frac{2 \sqrt{2 \ln 2}}{\ln ^{2} 2},-\frac{3}{2}, \frac{1}{3}, \frac{5}{2}, \frac{2 \sqrt{2 \ln 2}}{\ln ^{2} 2}\right\}\right\}$ Si el estudiante bosqueja lo siguiente: $\mathbb{R} \cap S$ y excluye de la intersección: $\left\{-\frac{2 \sqrt{2 \ln 2}}{\ln ^{2} 2},-\right.$ $\left.\frac{3}{2}, \frac{1}{3}, \frac{5}{2}, \frac{2 \sqrt{2 \ln 2}}{\ln ^{2} 2}\right\}$, se formarán ocho intervalos $A_{i}$ unidos que representan otra forma de notar el dominio de la función $\mathrm{g}(\mathrm{x}): \mathrm{A}_{1}=\left(-\infty,-\frac{2 \sqrt{2 \ln 2}}{\ln ^{2} 2}\right), \mathrm{A}_{2}=\left(-\frac{2 \sqrt{2 \ln 2}}{\ln ^{2} 2},-\frac{3}{2}\right), \mathrm{A}_{3}=\left(-\frac{3}{2},-\frac{\sqrt{2}}{2}\right.$. $\sqrt{\sqrt{5}-1}], \mathrm{A}_{4}=\left[0, \frac{1}{3}\right) \quad, \quad \mathrm{A}_{5}=\left(\frac{1}{3}, \frac{\sqrt{2}}{2} \cdot \sqrt{\sqrt{5}-1}\right], \mathrm{A}_{6}=\left[1, \frac{5}{2}\right), \quad \mathrm{A}_{7}=\left(\frac{5}{2}, \frac{2 \sqrt{2 \ln 2}}{\ln ^{2} 2}\right), \mathrm{A}_{8}=\left(\frac{2 \sqrt{2 \ln 2}}{\ln ^{2} 2}, \infty\right)$. Finalmente:

$$
\mathrm{D}(\mathrm{g}(\mathrm{x}))=\mathrm{A}_{1} \cup \mathrm{A}_{2} \cup \mathrm{A}_{3} \cup \mathrm{A}_{4} \cup \mathrm{A}_{5} \cup \mathrm{A}_{6} \cup \mathrm{A}_{7} \cup \mathrm{A}_{8}=\bigcup_{\mathrm{i}=1}^{8} \mathrm{~A}_{\mathrm{i}}
$$

Note que, en este tipo de análisis, uno de los intereses del estudiante debe ser el hallazgo de las raíces de una función y las constantes causantes de indeterminación, esto con el fin de analizar qué sucede en estos puntos que pueden ser llamados, para este contexto, valores críticos.

\subsubsection{Rango de la función $g(x)$}

Calcular el rango de una función no siempre es tarea fácil, puesto que el tratamiento adicional que este proceso requiere y como se mencionó en el título 3.2.2, se debe expresar la variable independiente en términos de la variable dependiente, para una función como $\mathrm{g}(\mathrm{x})$ tal hallazgo no es posible, al menos, con los recursos que la matemática fundamental brinda, esto no quiere decir que el dominio se pueda hallar fácilmente para cualquier función, inclusive no lo es, puesto que se ha notado que para obtener intervalos de solución y hallar valores causantes de indeterminación, es necesario encontrar las raíces de los polinomios o expresiones trascendentes 
que participan en la función, lo cual no siempre resulta sencillo, para ello es necesario que el estudiante después de tener claras las nociones y análisis que hasta aquí se han presentado, en compañía de los estudios subsiguientes al Cálculo y áreas esenciales afines, empiece a profundizar en lo que en matemáticas se denomina como: Análisis numérico, consúltese como referencia base (BURDEN; FAIRES, 2011), en este campo, empezará a conocer los distintos algoritmos que existen para calcular raíces por métodos no convencionales (algunos de ellos como el método de bisección, Newton, Horner, entre otros) o modelar funciones no elementales por medio de interpolación, entre otras características que esta línea de estudio y de investigación proporciona.

\subsection{Función trascendental}

El siguiente caso consiste en una función trigonométrica compuesta de una expresión logarítmica, hace parte de las funciones trascendentes, al igual que las exponenciales. En el título 4.1 se introdujo la trascendencia con el factor $\mathrm{u}_{4}(\mathrm{x})$, ahora se determinará el dominio y rango de la función $h(\mathrm{t})$ :

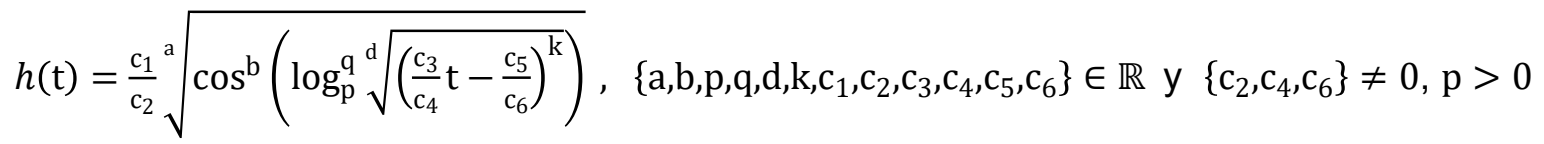

Es necesario reescribir $h(\mathrm{t})$ para comprender de una mejor forma el análisis:

$$
h(t)=\frac{c_{1}}{c_{2}} \sqrt{\left[\cos \left(\log _{p}\left(\frac{c_{3}}{c_{4}} t-\frac{c_{5}}{c_{6}}\right)^{\frac{k}{d}}\right)^{q}\right]^{b}}=\frac{c_{1}}{c_{2}} \sqrt{\left[\cos \left(\frac{k}{d} \log _{p}\left(\frac{c_{3}}{c_{4}} t-\frac{c_{5}}{c_{6}}\right)\right)^{q}\right]^{b}}
$$

Sea $\delta=\frac{c_{3}}{c_{4}} t-\frac{c_{5}}{c_{6}}, \beta=\left(\frac{k}{d} \log _{p} \delta\right)^{q}$ y $\varphi=\log _{p} \delta$. La función coseno $h(t)$ se compone de una función logarítmica para la cual se debe cumplir que $\delta>0$, puesto que $\nexists ! \varphi \mid \mathrm{p}^{\varphi} \leq 0$, siendo $\delta=\mathrm{p}^{\varphi}$, de esta manera $\frac{c_{3}}{c_{4}} t-\frac{c_{5}}{c_{6}}>0$, y si $\frac{c_{3}}{c_{4}}>0$ entonces $t>\frac{c_{4} c_{5}}{c_{3} c_{6}}$ o si $\frac{c_{3}}{c_{4}}<0$, entonces $t<\frac{c_{5} c_{4}}{c_{6} c_{3}}$, sin embargo, esto solo introduce el análisis, debido a que $\beta$ se encuentra en función del coseno: $h(\mathrm{t})=$ $\frac{c_{1}}{c_{2}} \sqrt[a]{(\cos \beta)^{b}}$, a y b son constantes que generan las siguientes restricciones:

i) Si b > $0 \wedge$ a es par $\rightarrow(\cos \beta)^{b} \geq 0$;

ii) Si b $<0 \wedge$ a es par $\rightarrow\left(\frac{1}{\cos \beta}\right)^{b} \geq 0 \wedge(\cos \beta)^{b} \neq 0$;

iii) Si b $<0 \wedge$ a es impar $\rightarrow(\cos \beta)^{b} \neq 0$;

iv) Si $b=-a \rightarrow \cos \beta \neq 0$.

v) Existen otros casos como: $b>0$ y que a sea impar o que $b=a$, para los cuales de manera global $\cos \beta$ no tiene asociada una implicación, sin embargo, se sabe que $\beta$ equivale a $\left(\frac{\mathrm{k}}{\mathrm{d}} \log _{\mathrm{p}} \delta\right)^{\mathrm{q}}$, por lo tanto, para estos dos casos la implicación será como ya se definió, es decir, que 
se cumpla que $\delta>0$. En caso de que $\mathrm{b}=0$ siendo $\mathrm{a} \neq 0$ y considerando que $\beta$ cumple las condiciones lógicas para que sea válida, entonces el dominio de la función será el conjunto de los números reales, puesto que $h(\mathrm{t})$ se convierte en una función constante de la forma $h(\mathrm{t})=\frac{\mathrm{c}_{1}}{\mathrm{c}_{2}}, \mathrm{c}_{2} \neq$ 0. Note que si a es negativa o no negativa, de igual forma se generarán algunas de las restricciones ya descritas, por otro lado, $(\cos \beta)^{\mathrm{b}} \geq 0$ y $(\cos \beta)^{\mathrm{b}} \neq 0$ pueden ser reescritas como: $(\cos \beta)^{b} \geq 0 \rightarrow \cos \beta \geq 0,(\cos \beta)^{b} \neq 0 \rightarrow \cos \beta \neq 0$.

\subsubsection{Análisis de las restricciones i y ii para el cálculo de $D(h(t))$}

Las desigualdades trigonométricas deben analizarse desde una perspectiva distinta a las polinómicas, debido a que en ellas intervienen ángulos que dependen de la expresión trigonométrica en cuestión y que según su naturaleza deben cumplir ciertas características, por ejemplo, para $\cos \beta \geq 0$, el estudiante debe preguntarse ¿Para qué ángulos de $\beta$ se cumple que su coseno sea mayor o igual a cero?, En la Figura 3 se puede observar con ayuda de la circunferencia unitaria que, esta desigualdad se cumple para los intervalos $\left[0, \frac{\pi}{2}\right]$ y $\left[\frac{3 \pi}{2}, 2 \pi\right]$ (tenga en cuenta que de manera genérica que $\cos \beta=\mathrm{x}_{0}$, para cualquier par $\left(\mathrm{x}_{0}, \mathrm{y}_{0}\right)$ en la circunferencia) como $\cos \beta$ es una función periódica, entonces los dos anteriores intervalos representan solo un par de los infinitos que existen tales que tal condición sea válida, por ejemplo, si el recorrido se inicia en sentido contrario a las manecillas del reloj, entonces entre $\left[0,-\frac{\pi}{2}\right]$ y $\left[-\frac{3 \pi}{2},-2 \pi\right], \cos \beta \geq$ 0 también es válida.

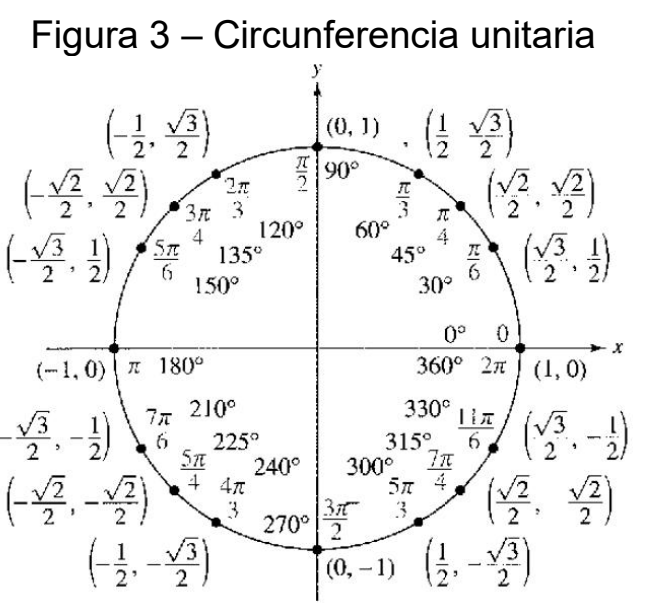

Fuente: Anexos del Libro de Cálculo I de Ron Larson, Hostetler y Edwards (2006, p. 812).

Por lo anterior, si se listan al menos cuatro intervalos consecutivos $\mathrm{I}_{\mathrm{i}}$ para los cuales la desigualdad se cumpla, se puede notar un patrón especial: $\mathrm{I}_{1}=\left[-\frac{\pi}{2}, \frac{\pi}{2}\right] ; \mathrm{I}_{2}=\left[\frac{3 \pi}{2}, \frac{5 \pi}{2}\right] ; \mathrm{I}_{3}=$ $\left[\frac{7 \pi}{2}, \frac{9 \pi}{2}\right] ; \mathrm{I}_{4}=\left[\frac{11 \pi}{2}, \frac{13 \pi}{2}\right]$. Los extremos de cada intervalo presentan un comportamiento lineal, semejante a una sucesión monótona creciente en donde cada término $a_{n}$ se comporta de la forma: 
$\mathrm{a}_{1}<\mathrm{a}_{2}<\mathrm{a}_{3} \cdots<\mathrm{a}_{\mathrm{n}-1}<\mathrm{a}_{\mathrm{n}}$, de esta manera se puede hallar el término n-ésimo $\mathrm{a}_{\mathrm{n}}$ y se puede afirmar que la forma en cómo se comportan ambos extremos obedecen a que $a_{n}=f_{n} \cdot n+d$, donde $f_{n}=\frac{a_{2}-a_{1}}{n_{2}-n_{1}}$ y d es un factor constante. Note que $f_{n} \cdot n+d$ es semejante a la fórmula general que modela una función lineal $y=m x+b$, de manera precisa el objetivo consiste en explotar esta noción haciendo una semejanza, sin asociar que $\mathrm{f}_{\mathrm{n}} \mathrm{y}$ d son congruentes conceptualmente con $\mathrm{m}$ y b . Para el extremo izquierdo de los $\mathrm{I}_{\mathrm{i}}$ la sucesión contiene términos como $\{\cdots,-$ $\left.\frac{\pi}{2}, \frac{3 \pi}{2}, \frac{7 \pi}{2}, \frac{11 \pi}{2}, \cdots a_{n_{i z q}}\right\}$ los cuales se pueden agrupar en pares de la forma $\left(n_{i}, a_{n_{i z q}}\right)$ donde $n_{i}$ representa una variable contadora de cada término de la sucesión de $\mathrm{a}_{\mathrm{n}_{\mathrm{izqu}}}$, por tanto, cada par asociado a los cuatro extremos izquierdos de los $I_{i}$ planteados son: (0, $\left.\frac{\pi}{2}\right),\left(1, \frac{3 \pi}{2}\right),\left(2, \frac{7 \pi}{2}\right),\left(3, \frac{11 \pi}{2}\right)$. Al escoger cualesquiera parejas consecutivas de las anteriores, para este caso $\left(0,-\frac{\pi}{2}\right),\left(1, \frac{3 \pi}{2}\right)$ se tiene que $\mathrm{f}_{\mathrm{n}_{\mathrm{izqu}}}=\frac{\frac{3 \pi}{2}-\left(-\frac{\pi}{2}\right)}{1-0}=\frac{\frac{4 \pi}{2}}{1}=2 \pi$. Hallando $\mathrm{d}$ y tomando como referente el par $\left(0,-\frac{\pi}{2}\right)$ para evaluar: $f_{n} \cdot n+d=a_{n_{\text {izqu }}}, \quad d=a_{n_{\text {izqu }}}-f_{n} \cdot n, d=-\frac{\pi}{2}-(2 \pi)(0)=-\frac{\pi}{2}$. Por lo tanto $\mathrm{a}_{\mathrm{n}_{\mathrm{izq}}}=2 \pi \mathrm{n}-\frac{\pi}{2}$. Si se realiza el mismo análisis para la sucesión de términos que se forman por el extremo derecho de cada intervalo se obtiene: $a_{n_{\text {der }}}=2 \pi n+\frac{\pi}{2}$, por tanto, $\cos \beta \geq$ $0 \leftrightarrow \beta \in\left[2 \pi n-\frac{\pi}{2}, 2 \pi n+\frac{\pi}{2}\right], \forall n \in \mathbb{Z}$. Para ambos extremos respecto a $\beta=2 \pi n \pm \frac{\pi}{2}$. Tenemos que $\left(\frac{\mathrm{k}}{\mathrm{d}} \log _{\mathrm{p}}\left(\frac{\mathrm{c}_{3}}{\mathrm{c}_{4}} \mathrm{t}-\frac{\mathrm{c}_{5}}{\mathrm{c}_{6}}\right)\right)^{\mathrm{q}}=2 \pi \mathrm{n} \pm \frac{\pi}{2} \therefore \mathrm{t}=\frac{\mathrm{c}_{4}}{\mathrm{c}_{3}}\left(\mathrm{p}^{\frac{\mathrm{d}}{\mathrm{k}} \sqrt{2 \pi \mathrm{n} \pm \frac{\pi}{2}}}+\frac{\mathrm{c}_{5}}{\mathrm{c}_{6}}\right)$. Para $h(\mathrm{t})$, cumpliéndose la restricción i, el dominio $\mathrm{D}(h(\mathrm{t}))$ es equivalente a:

$$
\mathrm{D}(h(\mathrm{t}))=\left\{\mathrm{t} \in \mathbb{R} \mid \mathrm{t} \in\left[\frac{\mathrm{c}_{4}}{\mathrm{c}_{3}} \cdot\left(\mathrm{p}^{\frac{\mathrm{d} \mathrm{q}}{\mathrm{k}} \sqrt{2 \pi n-\frac{\pi}{2}}}+\frac{\mathrm{c}_{5}}{\mathrm{c}_{6}}\right), \frac{\mathrm{c}_{4}}{\mathrm{c}_{3}} \cdot\left(\mathrm{p}^{\frac{\mathrm{d}}{\mathrm{k}} \sqrt{2 \pi n+\frac{\pi}{2}}}+\frac{\mathrm{c}_{5}}{\mathrm{c}_{6}}\right)\right] \wedge\left\{\mathrm{k}, \mathrm{c}_{3}, \mathrm{c}_{6}\right\} \neq 0, \forall \mathrm{n} \in \mathbb{Z}\right\}
$$

Sujeto a que si $q$ es par, entonces $n \geq \frac{1}{4}$ (es necesario aclararlo aunque $n \in \mathbb{Z}$ ) y para cualquier restricción, si $\frac{c_{3}}{c_{4}}>0$ entonces $t>\frac{c_{5} c_{4}}{c_{6} c_{3}}$ o si $\frac{c_{3}}{c_{4}}<0$, entonces $t<\frac{c_{5} c_{4}}{c_{6} c_{3}}$. Respecto a la restricción ii, se tiene que $\left(\frac{1}{\cos \beta}\right)^{\mathrm{b}} \geq 0$, esta desigualdad solo se cumplirá si $\cos \beta>0$, aclarando que $\left(\frac{1}{\cos \beta}\right)^{\mathrm{b}}$ nunca será igual a cero. $\cos \beta>0$ es similar a la restricción generada en i, por tanto, en términos de que los extremos del intervalo deben ser abiertos y no cerrados, adicional a esto, para ii se debe cumplir que $\cos \beta \neq 0$, esto quiere decir que se deben excluir todos los valores de $\mathrm{t}$ que en $\beta$ hacen que $\cos \beta=0$, aunque al resolver la desigualdad $\cos \beta>0$, automáticamente se excluyen los valores de $\beta$ para los cuales $\cos \beta=0$, no está demás mostrar la expresión general que modela cada dato. Algunos de los términos de $\beta$ para los cuales $\cos \beta=0$ son: $\left\{\cdots \frac{\pi}{2}, \frac{3 \pi}{2}, \frac{5 \pi}{2}, \frac{7 \pi}{2} \cdots a_{n}\right\}$ y al igual que en $\mathrm{i}$, cumplen un patrón lineal cuyo término $\mathrm{n}$-ésimo es $\mathrm{a}_{\mathrm{n}}=$ 
$\pi n+\frac{\pi}{2}$, por tanto $\beta \neq \pi n+\frac{\pi}{2}$ y se deduce que $t \neq \frac{c_{4}}{c_{3}}\left(p^{\frac{d}{k}} \sqrt{\pi n+\frac{\pi}{2}}+\frac{c_{5}}{c_{6}}\right)$. Finalmente, el dominio de la función para ii es:

$$
\mathrm{D}(h(\mathrm{t}))=\left\{\mathrm{t} \in \mathbb{R} \mid \mathrm{t} \in\left[\frac{\mathrm{c}_{4}}{\mathrm{c}_{3}} \cdot\left(\mathrm{p}^{\frac{\mathrm{d}}{\mathrm{k}} \sqrt{2 \pi n-\frac{\pi}{2}}}+\frac{\mathrm{c}_{5}}{\mathrm{c}_{6}}\right), \frac{\mathrm{c}_{4}}{\mathrm{c}_{3}} \cdot\left(\mathrm{p}^{\frac{\mathrm{d}}{\mathrm{k}} \sqrt{2 \pi n+\frac{\pi}{2}}}+\frac{\mathrm{c}_{5}}{\mathrm{c}_{6}}\right)\right] \wedge\left\{\mathrm{k}, \mathrm{c}_{3}, \mathrm{c}_{6}\right\} \neq 0, \forall \mathrm{n} \in \mathbb{Z}\right\}
$$

Sin olvidar las condiciones que cumple n si q es par. Aunque en $\mathrm{D}(h(\mathrm{t}))$ se escriba un solo intervalo genérico, esto no quiere decir que existe un solo conjunto válido, por el contrario, existen $\mathrm{m}$ conjuntos de intervalos válidos unidos para cada $\mathrm{n}$ consecutivo que pertenece $\mathrm{a} \mathbb{Z}$.

\subsubsection{Análisis para calcular $\mathrm{D}^{\prime}(\mathrm{h}(\mathrm{t}))$}

Se sabe que para hallar $\mathrm{D}^{\prime}(h(\mathrm{t}))$ es necesario expresar $\mathrm{t}$ en términos de $h(\mathrm{t})$, si $h(\mathrm{t})=\mathrm{y}$, entonces:

$$
\frac{c_{1}}{c_{2}} \cdot \sqrt[a]{\cos ^{b}\left(\log _{p} q \sqrt[d]{\left(\frac{c_{3}}{c_{4}} t-\frac{c_{5}}{c_{6}}\right)^{k}}\right)}=y \quad \therefore \quad t=\frac{c_{4}}{c_{3}}\left(\frac{\mathrm{d}}{\mathrm{k}} \cdot \sqrt[q]{\arccos \left(\sqrt{\left(\frac{c_{2}}{c_{1}} y\right)^{a}}\right)}+\frac{c_{5}}{c_{6}}\right)
$$

Fíjese que para t tambien se puede afirmar que $\mathrm{k}, \mathrm{c}_{1} \circ \mathrm{c}_{3}$ deben ser distintas de cero, sin embargo, si estas lo fuesen, al comienzo del problema la función automáticamente tomará otras formas, debido a que estas constantes en particular, si pueden tomar el valor de cero cuando $t \rightarrow$ $h(\mathrm{t})$. Por otro lado, se obtiene una función exponencial compuesta por una función trigonométrica inversa, de la cual se pueden resumir sus términos: $\alpha=\sqrt[b]{\left(\frac{c_{2}}{c_{1}} y\right)^{a}}, \mu=\arccos (\alpha), \sigma=\sqrt[q]{\mu}$. Si se analiza la implicación generada para $\mu$, se debe precisar que $\alpha \in[-1,1]$, esto se deduce en términos de que la pregunta que debe hacerse el estudiante es ¿Entre qué valores oscila cos $\mu$ ? Puesto que $\alpha=\cos \mu$, de esta manera se sabe que $\alpha$ pertenecerá a tal intervalo, así que si $\alpha=$ $\sqrt[b]{\left(\frac{c_{2}}{c_{1}} y\right)^{a}}$ entonces: $-1 \leq \sqrt[b]{\left(\frac{c_{2}}{c_{1}} y\right)^{a}} \leq 1$, por ende: $\frac{c_{1}}{c_{2}} \sqrt[a]{(-1)^{b}} \leq y \leq \frac{c_{1}}{c_{2}} \sqrt[a]{(1)^{b}}$, por lo que se puede afirmar que $y \in\left[\frac{c_{1}}{c_{2}} \sqrt[a]{(-1)^{b}}, \frac{c_{1}}{c_{2}} \sqrt[a]{(1)^{b}}\right]$, sin embargo, esta no puede ser una respuesta definitiva para el rango de $h(\mathrm{t})$, puesto que al igual que para el dominio, se deben analizar las restricciones que cumplen a y $\mathrm{b}$, sin olvidar que $\mu$ se supedita $a \sqrt[q]{\mu}$, para lo cual q también poseerá restricciones que hagan que $\mu \geq 0 \circ \mu \neq 0$, según corresponda. En estas se deben conocer los valores para los cuales $\alpha$ es válido en $\arccos (\alpha)$ y con el fin de promover la reflexión y/o análisis en el estudiante, este podrá proponer las distintas condiciones para las cuales $h(t)$ es consistente según esta última aclaración y discutir los resultados evaluando las constantes para números 
particulares. La idea radica en conservar la misma línea lógica para determinar el conjunto de valores válidos, analizando aquellos que no lo son.

\subsubsection{Otras consideraciones}

No todas las funciones trigonométricas poseen un dominio tal que en cada intervalo consecutivo exista periodicidad como lo fue en el caso de $h(\mathrm{t})$ tratada en el título 4.2.1, para funciones que poseen un estilo como: $s(x)=\frac{1}{2 \operatorname{sen} x+1}$. Tal periodicidad no se da en los extremos consecutivos de cada intervalo, como $2 \operatorname{sen} x+1 \neq 0$, para la cual $\operatorname{sen} x \neq-\frac{1}{2}$, algunos de los valores de $\mathrm{x}$ que hacen que senx $=-\frac{1}{2}$ y que por tanto deben ser excluidos del intervalo de solución de $\mathrm{D}(\mathrm{s}(\mathrm{x}))$ son: $\mathrm{x}=\left\{\cdots,-\frac{\pi}{6}, \frac{7 \pi}{6}, \frac{11 \pi}{6}, \frac{19 \pi}{6}, \frac{23 \pi}{6} \cdots\right\}$. Si se representa solo un fragmento de algunos de los infinitos intervalos para los cuales $\mathrm{s}(\mathrm{x})$ está definida, con base en lo anterior se tienen: $\left(-\frac{\pi}{6}, \frac{7 \pi}{6}\right) ;\left(\frac{7 \pi}{6}, \frac{11 \pi}{6}\right) ;\left(\frac{11 \pi}{6}, \frac{19 \pi}{6}\right) ;\left(\frac{19 \pi}{6}, \frac{23 \pi}{6}\right)$. Note que el patrón de linealidad para los extremos de cada intervalo no se da de forma consecutiva sino alternada, realizando el análisis descrito en pasos anteriores, el dominio de la función es:

$$
\mathrm{D}(\mathrm{s}(\mathrm{x}))=\left\{\mathrm{x} \in \mathbb{R} \mid \mathrm{x} \in\left(2 \pi \mathrm{n}-\frac{\pi}{6}, 2 \pi \mathrm{n}+\frac{7 \pi}{6}\right) \cup\left(2 \pi \mathrm{n}+\frac{7 \pi}{6}, 2 \pi \mathrm{n}+\frac{11 \pi}{6}\right), \mathrm{n} \in \mathbb{Z}\right\}
$$

Note también que $\mathrm{D}(\mathrm{s}(\mathrm{x}))$ se ha representado para cada pareja de intervalos consecutivos unidos en la cual se pueda evaluar cualquier $\mathrm{n}, \mathrm{D}(\mathrm{s}(\mathrm{x})$ ) también puede ser representado por medio de una rotación completa, es decir, fijar el par de intervalos descritos en $\mathrm{D}(\mathrm{s}(\mathrm{x})$ ) en el intervalo entre cero y $2 \pi$; En condiciones generales, como desee expresarlo el analista del problema o como le sea requerido, teniendo en cuenta que para algún $n$ se debe garantizar que los intervalos unidos resultantes sean válidos para $\mathrm{s}(\mathrm{x})$. Nótese que el cálculo del rango de $\mathrm{s}(\mathrm{x})$ para este caso es más sencillo, consiste en solucionar una desigualdad básica, puesto que al expresar $s(x) \rightarrow x=\operatorname{arcsen}\left(\frac{1-s(x)}{2 s(x)}\right)$. Para lo cual se debe solucionar $-1 \leq \frac{1-s(x)}{2 s(x)} \leq 1$ y se obtiene que $D^{\prime}(s(x))=(-\infty,-1] \cup\left[\frac{1}{3}, \infty\right) \quad V \quad D^{\prime}(s(x))=\mathbb{R}-\left(-1, \frac{1}{3}\right)$. Observe que al expresar $\mathrm{D}^{\prime}(\mathrm{s}(\mathrm{x}))=\mathbb{R}-\left(-1, \frac{1}{3}\right)$, el intervalo de exclusión, es decir $\left(-1, \frac{1}{3}\right)$ es abierto, puesto que si este se dejase cerrado, se da por entendido que ambos extremos no hacen parte del dominio de la función, lo cual no es correcto; Esta expresión de cierta forma es implícita, pues se deduce que al excluir $\left(-1, \frac{1}{3}\right)$, se obtiene $(-\infty,-1] \cup\left[\frac{1}{3}, \infty\right)$, la cual representa su forma explícita. 


\subsection{Otras funciones trascendentales}

Existen otras funciones clásicas de las cuales es necesario mencionar ciertas características, por ejemplo, funciones hiperbólicas, funciones valor absoluto o la función parte entera.

\subsubsection{Funciones hiperbólicas}

Son realmente casos particulares de funciones exponenciales, tienen la característica de poseer cierta semejanza con las funciones trigonométricas, no en fondo, sino en forma, en términos de que estas también poseen sus propias identidades, las cuales el estudiante no puede confundir por causa de la leve semejanza, por ejemplo, si se requiere determinar el dominio de una función hiperbólica compuesta de una función trigonométrica como:

$$
\mathrm{f}(\mathrm{x})=\frac{1}{\operatorname{sen} h^{2}(\operatorname{sen}(\gamma \mathrm{x}))+\cosh ^{2}(\operatorname{sen}(\gamma \mathrm{x}))}, \gamma \in \mathbb{Z}
$$

De la identidad trigonométrica fundamental se sabe que $\operatorname{sen}^{2} x+\cos ^{2} x=1$, sin embargo, para las funciones hiperbólicas $\operatorname{sen} h^{2}(\mathrm{x})+\cosh ^{2}(\mathrm{x}) \neq 1$, en algún texto de Cálculo (STEWART, 2012) el estudiante puede indagar en las identidades hiperbólicas, definiciones y demás, para demostrar que $\operatorname{senh}^{2}(\mathrm{x})+\cosh ^{2}(\mathrm{x})=\cosh (2 \mathrm{x})$, aparte de esto, el coseno hiperbólico se define como:

$$
\cosh \vartheta=\frac{\mathrm{e}^{\vartheta}+\mathrm{e}^{-\vartheta}}{2}
$$

Lo anterior aplicado en $f(x)$ se traduce como: $f(x)=\frac{1}{\cosh (2 \operatorname{sen}(\gamma x))}=\frac{2 e^{2 \operatorname{sen}(\gamma x)}}{e^{4 \operatorname{sen}(\gamma x)}+1}$, por lo que hasta aquí se ha profundizado, el estudiante puede deducir fácilmente que $\mathrm{D}(\mathrm{f}(\mathrm{x}))=\mathbb{R}$. Uno de los hechos es mostrar que también fácilmente se puede cometer el error y pensar que el denominador de $\mathrm{f}(\mathrm{x})$ es igual a uno, y decir que el dominio equivale a todo el conjunto de los números reales, para este caso, es casual llegar al mismo resultado, pero con un procedimiento errado por causa de un concepto mal definido. De la misma manera puede demostrar que: $\mathrm{x}=$ $\frac{1}{\gamma} \operatorname{arcsen}\left(\frac{1}{2} \ln \left(\frac{1 \pm \sqrt{1-\mathrm{f}^{2}(\mathrm{x})}}{\mathrm{f}(\mathrm{x})}\right)\right)$ y que por tanto $\mathrm{D}^{\prime}(\mathrm{f}(\mathrm{x}))=\left[\frac{2 \mathrm{e}^{4}}{\mathrm{e}^{2}\left(\mathrm{e}^{4}+1\right)}, 1\right]$. Note que $\gamma$ no posee restricciones desde $\mathrm{f}(\mathrm{x})$, y sin importar su valor en $\mathbb{R}$, tampoco posee implicaciones en su dominio o rango, inclusive, aunque $\gamma=0, \mathrm{f}(\mathrm{x})=1$.

\subsubsection{Funciones valor absoluto}

Son realmente funciones a trozo, en las cuales el estudiante debe tener clara su definición, y a partir de esta, el análisis empieza a ser similar a lo que hasta aquí se ha tratado y 
evidentemente dependiendo de la composición que la función valor absoluto posea. Si se plantea una función como: $\mathrm{f}(\mathrm{x})=\mid \frac{\mathrm{ax}{ }^{2}+\mathrm{bx}+\mathrm{c}}{\mathrm{dx^{2 } + \mathrm { kx } + \mathrm { t }} \mid},\{\mathrm{a}, \mathrm{b}, \mathrm{c}, \mathrm{d}, \mathrm{k}, \mathrm{t}\} \in \mathbb{R} \mathrm{d}, \mathrm{k} \mathrm{y}$ t no pueden ser simultáneamente cero.

Por su definición se sabe que: $\left|\frac{a x^{2}+b x+c}{d x^{2}+k x+t}\right|=\left\{\begin{array}{lll}\frac{a x^{2}+b x+c}{d x^{2}+k x+t} & \text { si } & \frac{a x^{2}+b x+c}{d x^{2}+k x+t} \geq 0 \\ -\left(\frac{a x^{2}+b x+c}{d x^{2}+k x+t}\right) & \text { si } & \frac{a x^{2}+b x+c}{d x^{2}+k x+t}<0\end{array}\right.$

Cuando la función se desglosa en sus dos funciones correspondientes, entonces el análisis se hará por partes y luego de manera global se analiza para qué valores de $\mathrm{x}$ es válida según cada condición solucionando las desigualdades. Respecto al rango, el estudiante debe resolver

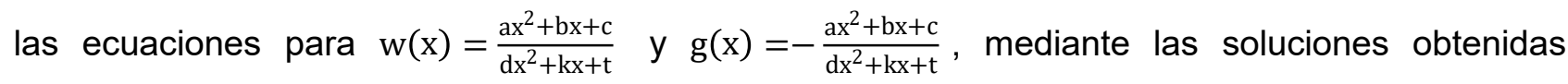
establecer los intervalos que cubren todos los valores posibles y válidos de $\mathrm{f}(\mathrm{x})$, que en ultimas, $\mathrm{f}(\mathrm{x})$ se compone de las funciones $\mathrm{w}(\mathrm{x})$ y $\mathrm{g}(\mathrm{x})$. Por otro lado, no siempre se debe pensar que todas las funciones de la formada $y=|p(x)|$ tiene como rango todos los reales positivos, no solo porque se deben solucionar las ecuaciones para las funciones a trozo que pertenecen a $|\mathrm{p}(\mathrm{x})|$, sino porque puede existir una transformación que haga que la función tome valores negativos en la variable independiente o positivos que no inician en $\mathrm{y}=0$, para observarlo de manera ligera por medio de una función sencilla como $\mathrm{y}=|\mathrm{x}|$, su rango es $\mathrm{D}^{\prime}(\mathrm{y})=[0, \infty)$, pero si $\mathrm{y}=|\mathrm{x}|+\mathrm{c}$, entonces $D^{\prime}(y)=[c, \infty)$.

\subsubsection{Función parte entera}

Son funciones que en ocasiones en las aulas de clase no se profundizan lo suficiente, pero hacen parte del análisis matemático, puesto que desde estas el estudiante puede empezar a entender la noción de transformación, en términos de que esta función toma un número cualesquiera $\mathrm{x}$ que pertenece al conjunto de los números reales y entrega ya sea el máximo entero $\mathrm{n}$ no superior $\mathrm{a} x$ o el mínimo entero $\mathrm{n}$ no inferior a $\mathrm{x}$. Para el primer caso la función se denomina clásicamente como función techo y se expresa como $\mathrm{y}=\lceil\mathrm{x}\rceil$, de la misma forma, para el segundo caso la función se denomina como función piso y se expresa como $y=\lfloor x\rfloor$. Por ejemplo: $\lceil 0\rceil=0 ;[0.6\rceil=1 ;\lceil-2.4\rceil=-2 ;\lfloor-1.6\rfloor=-2 ;\lfloor-4\rfloor=-4 ;[4.5\rfloor=4$. Es necesario que el estudiante tenga interacción gráfica con la función, esto con el fin de tener una visión más amplia de la misma, inclusive, se pueden modelar en softwares matemáticos didácticos como GeoGebra (CARABALLO; GONZÁLEZ, 2015) y posteriormente entender bien sus propiedades (CASTIBLANCO ACOSTA, 2013). Estas funciones también pueden combinarse con otras que no son necesariamente de esta naturaleza, por ejemplo: $y=[x]+\frac{x}{2}$, que contiene una función techo $\mathrm{y}_{1}(\mathrm{x})=\lceil\mathrm{x}\rceil \mathrm{y}$ la función lineal $\mathrm{y}_{2}(\mathrm{x})=\frac{\mathrm{x}}{2}$, en este orden de ideas, un sector de la representación gráfica de y es: 
Figura 4 - Representación Gráfica de una función parte entera combinada con una función lineal

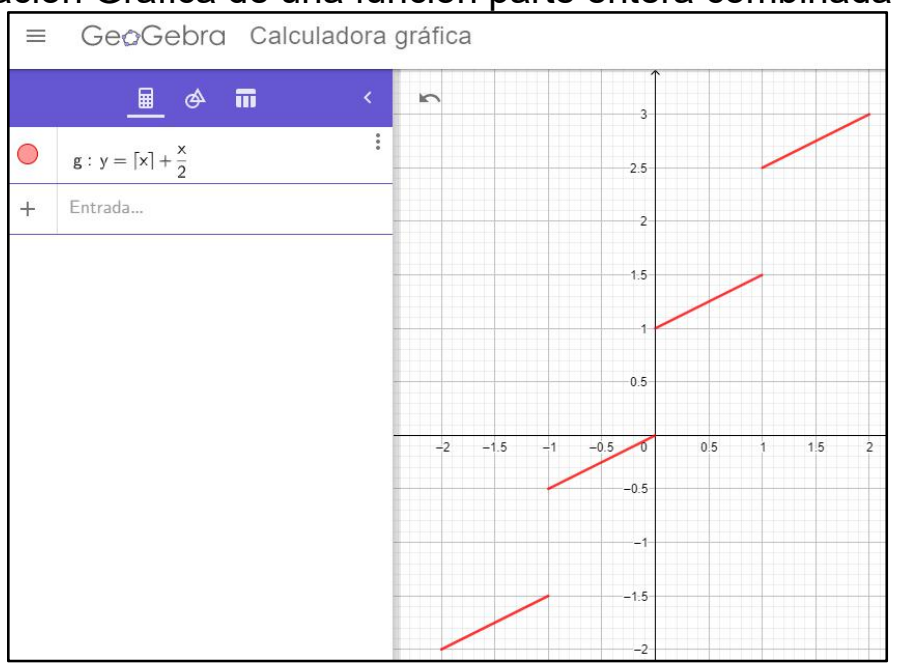

Fuente: Elaborado en GeoGebra.

Esta función tiene una característica especial que consiste en el análisis de la continuidad para cada valor entero, como la Figura 4 no expresa de forma clara tal continuidad, es necesario precisar que cada entero que hace parte del dominio de la función no posee dos imágenes sino solo una, por ejemplo, $y(1)=\frac{3}{2}$, aunque $\lim _{x \rightarrow 1^{+}}\left(\lceil x\rceil+\frac{x}{2}\right)=\frac{5}{2}$ y $\lim _{x \rightarrow 1^{-}}\left(\lceil x\rceil+\frac{x}{2}\right)=\frac{3}{2}$, no obstante, la función no es continua en $x=1$, principalmente porque $\lim _{x \rightarrow 1}\left(\lceil x\rceil+\frac{x}{2}\right)=\nexists$, aun así, para $x=1$, y se encuentra definida. Este mismo análisis se debe replicar para todos los enteros de $\mathrm{x}$. El dominio de la función se define para todos los números reales $\mathrm{D}(\mathrm{y})=\mathbb{R}$. Note que el rango al igual que en las funciones trigonométricas debe ser expresado en forma de término enésimo de una sucesión, pues está definido para intervalos como $\left(-\frac{1}{2}, 0\right],\left(1, \frac{3}{2}\right],\left(\frac{5}{2}, 3\right]$, entre otros infinitos que se expresan como: $D^{\prime}(y)=\left\{y \in \mathbb{R} \mid y \in\left(\frac{3}{2} \mathrm{n}-\frac{1}{2}, \frac{3}{2} \mathrm{n}\right], \mathrm{n} \in \mathbb{Z}\right\}$. Note que para este último ejemplo, con el fin de aclarar asuntos gráficos, fue necesario acudir al análisis de continuidad para un punto específico, y esto no solo aplica para este tipo de funciones, sino que para cientos de funciones es necesario realizar este análisis, puesto que a simple vista en muchas ocasiones se pueden cometer errores, que pueden considerarse al excluir del dominio valores para los cuales la variable independiente o dependiente si estén definidos, o incluso, que suceda lo contrario. Esto depende del grado de complejidad de la función y por tanto la facilidad que exista para descomponerla en factores que hagan el análisis más ligero. Con todo y esto, se puede fijar cuán importante es emplear las técnicas provenientes de la matemática fundamental, para funciones que a simple vista parecen ser tener un tratamiento matemático que demanda técnicas avanzadas, pero en realidad, solo se debe analizar la función con detenimiento y establecer una serie de condiciones que tracen un camino que despeje concienzudamente el hallazgo de la solución. 


\section{Comentarios finales}

En este artículo se introdujo que existen relaciones entre ciertos conjuntos y otros, pero esto no delimita a la matemática pura como la ciencia única que pueda establecer tales relaciones, y que por tanto los estudiantes van a encontrarlas solo en sus primeros cursos de Cálculo. Por el contrario, tales relaciones se expanden hacia diversos campos de aplicación como la física o la ingeniería, en las que muchos profesionales de diversas disciplinas utilizan relaciones para modelar o identificar situaciones reales, por ejemplo, el análisis de señales análogas en el caso de la electrónica, determinación de procesos politrópicos en el campo de la termodinámica, problemas logísticos en el caso de la investigación operativa, o las funciones de densidad utilizadas en el análisis probabilístico. Lo anterior se trae a colación, debido a que los ejemplos aquí tratados, son extensiones de funciones o relaciones que presentan configuraciones similares en modelos (CRUZ HUERTAS; MEDINA CASTAÑEDA, 2013) planteados que sitian la vida real, en los cuales, aparte de tener en cuenta el contexto conceptual sobre el cual se basa la modelación, se debe precisar que tal modelo se compone de variables que no pueden tomar cualquier valor aleatorio y que por el contrario se sesgan debido al alcance que tienen en el conjunto de los números reales; en otras palabras, están sujetas ya sea a un conjunto de restricciones definidas por la función misma o la conceptualización que rodea su construcción. Por ejemplo, si un estudiante que está especializándose en la rama de las Finanzas Cuantitativas, entre tantas ecuaciones con las cuales puede toparse en su actividad académica, analiza la fórmula de Black-Scholes (VILLAMIL, 2006), inmediatamente identifica que esta contiene factores trascendentes. Independientemente que tales factores sean variables o no, puede determinar el conjunto de valores para los cuales estos son válidos en $\mathbb{R}$, entenderá en un primer plano la razón por la cual cualquier indicador financiero o factor específico de la formula, puede tomar ciertos valores en un rango específico. La idea consiste en que el análisis del dominio y rango se expanda no solo para el tratamiento puro de las matemáticas, sino que se explote al interior de las aplicaciones empleadas en ciencias e ingeniería, pues, a fin de cuentas, la gran mayoría de las actividades que embarcan nuestro entorno, son traducidas a expresiones matemáticas compuestas de variables y parámetros que cumplen ciertas condiciones según su configuración y contexto planteado y que por tanto tal composición será válida para un intervalo especial de números.

\section{Referencias}

ADAMS, R. A. Cálculo. 6. ed. Madrid: Addison Wesley, 2009. 1230 p.

APOSTOL, T. M. Cálculo con funciones de una variable, con una introducción al álgebra lineal. v. 1. 2. ed. Barcelona: Reverté, 1984. 836 p. 
BURDEN, R. L.; FAIRES, J. D. Numerical Analysis. Canada: Editorial Brooks Cole Cengage Learning, 2011.

CARABALLO, H.; GONZÁLEZ, C. Z. Aplicación de funciones de parte entera. Objeto de enseñanza. In: CONGRESO SOBRE TECNOLOGÍA EN EDUCACIÓN \& EDUCACIÓN EN TECNOLOGÍA, 10., 11 y 12 jun. 2015, Corrientes. Actas [...]. [S. I.]: [s. n.], 2015. p. 520-527. Disponible: http://sedici.unlp.edu.ar/bitstream/handle/10915/49095/Documento completo.pdf?sequence=1\&is Allowed=y. Acceso en: 29 enero 2022.

CASTIBLANCO ACOSTA, M. A. Función Parte Entera desde la Modelación. In: CONGRESO IBEROAMERICANO DE EDUCACIÓN MATEMÁTICA, 7., 16 al 20 sept. 2013, Montevideo, Uruguay. Actas [...]. [S. I.]: [s. n.], 2013. p. 1187-1194. Disponible:

http://funes.uniandes.edu.co/19975/1/Castiblanco2013Funci\%C3\%B3n.pdf. Acceso en: 29 enero 2022.

CEBALLOS URREGO, L.; LÓPEZ MONSALVE, A. Relaciones y Funciones: conceptos clave para el aprendizaje del cálculo, y una propuesta para la aplicación del modelo de Van Hiele. Educación y Pedagogía, [s. I.], v. 15, n. 35, p. 131-140, 2003. Disponible: https://revistas.udea.edu.co/index.php/revistaeyp/article/view/5948. Acceso en: 1 dic. 2021.

CRIBEIRO, J. M.; MADRID, H.; FRAGA, J. L. ¿Relación, función o ecuación? EI Cálculo y su Enseñanza, Cinvestav, México, v. 5. año 5, p. 41-56, sept. 2013/sept. 2014.

CRUZ HUERTAS, J.; MEDINA CASTAÑEDA, Y. Funciones en contexto. Una experiencia enriquecida en la modelación y simulación interactiva. Sistemas \& Telemática, Cali, v. 11, n. 26, p. 59-80, 30 sept. 2013. DOI: http://dx.doi.org/10.18046/syt.v11i26.1629.

GAMBOA, A. R.; CASTILLO, S. M.; HIDALGO MORA, R. Errores matemáticos de estudiantes que ingresan a la universidad. Actualidades Investigativas en Educación, San José, Costa Rica, v. 19, n. 1, 1 enero 2019. DOI: https://doi.org/10.15517/aie.v19i1.35278.

GOODMAN, A.; HIRSCH, L. Álgebra y Trigonometría con Geometría Analítica. 1. ed. Naucalpan de Juarez: Prentice Hall Hispanoamericana, 1996.

LEITHOLD, L. El Cálculo. 7. ed. México: Oxford University Press, Harla México, 1998. 1382 p.

LUPO, L. Dominio de funciones matemáticas en estudiantes de ingeniería de la Universidad Católica Andrés Bello. Revista ORBIS, [s. I.], v. 1, n. 2, p. 4-24, nov. 2005. Disponible: http://www.revistaorbis.org/pdf/2/2Art1.pdf. Acceso en: 29 enero 2022.

MEJÍA PALOMINO, M. F. La factorización de polinomios de una variable real en un ambiente de lápiz/papel (L/P) y álgebra computacional (CAS). Directores: Diego Garzón Castro; Jorge Hernando Arce Chaves. 2011. 309 f. Tesis (Maestía en Educación con énfasis en Educación Matemática) - Universidad del Valle, Santiago de Cali, 18 oct. 2011. Disponible: http://hdl.handle.net/10893/4079. Acceso en: 1 dic. 2021.

OLIVAR MOLINA, S. A.; FLORES LÓPEZ, W. O.; ALVARADO GONZÁLEZ, F. D. Errores algebraicos en tareas de descomposición factorial por estudiantes universitarios de Nicaragua. Revista electrónica de conocimientos, saberes y prácticas, [s. I.], v. 1, n. 1, p. 9-27, 18 oct. 2018. DOI: https://doi.org/10.30698/recsp.v1i1.1. 
OOSTRA, A. Sobre la solución de ecuaciones de tercer y cuarto grado. Revista Tumbaga, [s. I.], v. 3, p. 174-186, 2008.

PÉREZ GONZÁLES, F. J. Cálculo diferencial e integral de funciones de una variable. Granada: [s. n.], 2008. 683 p.

PÉREZ LLUBERES, K. Álgebra Superior. v. 1. Santo Domingo, D. N.: Orto-Taller Dominicano C Por A, 1984. 392 p.

PÉREZ RAPOSO, Á. Lógica, conjuntos, relaciones y funciones. v. 12. [S. I.]: Publicaciones Electrónicas Sociedad Matemática Mexicana, Universidad Autónoma de San Luis Potosí, Universidad Politécnica de Madrid, 2010. 99 p.

PRADA NUÑEZ, R.; HERNÁNDEZ SUÁREZ, C. A.; RAMÍREZ LEAL, P. Comprensión de la noción de función y la articulación de los registros semióticos que la representan entre estudiantes que ingresan a un programa de ingeniería. Revista Científica CIDC, [s. I.], v. 25, n. 2, p. 188-205, 31 agosto 2016. DOI: https://doi.org/10.14483//udistrital.jour.RC.2016.25.a3.

LARSON, R.; HOSTETLER, R. P.; EDWARDS, B. H. Cálculo con geometría analítica. v. I. 8. ed. México: McGraw-Hill Interamericana, 2006.

ROUMIEU, S. M. La importancia de las funciones en la formulación de modelos matemáticos utilizando tecnología: implementación del modelo 1 a 1. In: CONGRESO IBEROAMERICANO DE CIENCIA, TECNOLOGÍA, INNOVACIÓN Y EDUCACIÓN. 12, 13 y 14 nov. 2014, Buenos Aires.

Actas [...]. [S. I.]: [s. n.], 2014. p. 1-14.

SANCHEZ GONZÁLEZ, E.; CANTÚ QUINTANILLA, A.; CANTÚ CUENA, Á. Concepciones de los estudiantes sobre dominio y rango de una función. In: CONGRESO INTERNACIONAL DE EDUCACIÓN: CURRÍCULUM, 29 y 30 sept. y 1 octubre 2011, Monterrey, México. Memorias [...]. [S. I.]: [s. n.], 2011. p. 1-7.

STEWART, J. Cálculo de una variable Trascendentes tempranas. 7. ed. México, D. F: Cengage Learning, 2012.

VALLE CASTAÑEDA, W.; CASTILLO ESTRELLA, T.; CAMEJO PUENTES , M. La evolución del aprendizaje del concepto función en la asignatura Matemática I. Revista de Educación MENDIVE, [s. I.], v. 16, n. 2, p. 222-237, abr./jun. 2018. Disponible:

http://scielo.sld.cu/scielo.php?script=sci arttext\&pid=S1815-76962018000200222. Acceso en: 29 enero 2022.

VANEGAS MACÍAS, D.; ESCALONA FUENMAYOR, M. Concepciones sobre funciones matemáticas de una variable, en estudiantes del primer semestre de ingeniería. Omnia, [s. I.], v. 19, n. 1, p. 99-113, enero/abr. 2013. Disponible: https://biblat.unam.mx/pt/revista/omniamaracaibo/articulo/concepciones-sobre-funciones-matematicas-de-una-variable-en-estudiantesdel-primer-semestre-de-ingenieria. Acceso en: 1 dic. 2021.

VILLAMIL, J. Modelos de valoración de opciones europeas en tiempo continuo. Cuadernos de Economía, Bogotá, v. 25, n. 44, jun. 2006. Disponible: http://www.scielo.org.co/scielo.php?script=sci arttext\&pid=S0121-47722006000100008. Acceso en: 1 dic. 2021. 